\title{
FILTERING A NONLINEAR SLOW-FAST SYSTEM WITH STRONG FAST FORCING*
}

\author{
BORIS GERSHGORIN ${ }^{\dagger}$ AND ANDREW MAJDA M $^{\ddagger}$
}

\begin{abstract}
A three-mode nonlinear slow-fast system with fast forcing is studied here as a model for filtering turbulent signals from partial observations. The model describes the interaction of two externally driven fast modes with a slow mode through catalytic nonlinear coupling. The special structure of the nonlinear interaction allows for the analytical solution for the first and second order statistics even with fast forcing. These formulas are used for testing the exact Nonlinear Extended Kalman Filter for the slow-fast system with fast forcing. Various practical questions such as the influence of the strong fast forcing on the slowly varying wave envelope, the role of observations, the frequency and variance of observations, and the model error due to linearization are addressed here.
\end{abstract}

Key words. Nonlinear model, slow-fast system, extended Kalman filter, fast forcing.

AMS subject classifications. 34A05, 93E11.

\section{Introduction}

The growing need for fast and accurate weather and climate prediction demands computationally inexpensive and effective filtering strategies, most of which are based on the classical Kalman Filter (KF) $[1,5,7,14,6,10,11,2,3,27,4,15,21]$. Filtering incorporates the dynamical forecast of the signal with full or partial observations in order to obtain the best least-squares approximation of the true signal. The dynamics of the studied signals is turbulent, with multiple time scales. A typical example is an atmosphere model where a slow advective vortical Rossby wave is nonlinearly coupled with the fast inertia-gravity waves [28, 9, 20]. A more complicated situation occurs in the tropics when the intermediate mixed Rossby-gravity and Kelvin waves are also present [29]. Moreover, the effect of tropical moist convection shows up as a strong forcing of the gravity waves [18, 24, 25, 30, 31, 32]. The bursts of strong fast forcing occur spontaneously and only last for short times. Moist convection that drives the fast gravity waves has a drastic effect on the dynamics of the atmosphere and, therefore, consideration of models that incorporate fast forcing is crucial for the problems of medium-range weather prediction. Filtering of atmospheric signals plays an important role in weather forecasting. How do the fast modes influence the slow dynamics, which is given by both the slow advective wave and the slowly varying envelope of the fast gravity waves? This is one of the central questions of filtering slow-fast systems. Another important issue for filtering atmospheric signals is that observations of the quantities such as temperature, pressure, or velocity mix slow and fast waves. Very often, one is faced with situations when the number of available observations is much smaller than the number of the state variables in the system: the partial observations case $[7,6,20]$. Moreover, most models that describe the atmosphere and ocean dynamics contain model errors due to inappropriate parametrization.

In this paper, we study a nonlinear three-dimensional multiple time test model with strong fast forcing. This model was first introduced in [13] where filtering strategies of the slow-fast systems were studied in detail. One of the important properties

\footnotetext{
${ }^{*}$ Received: July 7, 2008; accepted: September 24, 2008.

${ }^{\dagger}$ Dept. of Mathematics and Center for Atmosphere Ocean Science, Courant Institute of Mathematical Sciences, New York University (borisg@cims.nyu.edu).

${ }^{\ddagger}$ Dept. of Mathematics and Center for Atmosphere Ocean Science, Courant Institute of Mathematical Sciences, New York University (jonjon@cims.nyu.edu).
} 
of the present test model that was omitted in [13] is the presence of the strong fast forcing that adds effects mimicking moist convection in the model. The advantage of using this low dimensional test model for filtering is that on the one hand it is simple enough to have exactly solvable first and second order statistics needed for the dynamic forecast in the Nonlinear Extended Kalman Filter (NEKF). On the other hand, the test model carries important properties of the realistic systems such as multiple time scales, nonlinear non-Gaussian dynamics, and strong fast forcing.

The test model [13] is given by a three-dimensional system of stochastic differential equations for the slow real mode $u_{1}$ and complex fast mode $u_{2}$

$$
\begin{aligned}
& d u_{1}=\left(-\gamma_{1} u_{1}+f_{1}(t)\right) d t+\sigma_{1} d W_{1}, \\
& d u_{2}=\left(\left(-\gamma_{2}+i \omega_{0} / \varepsilon+i a_{0} u_{1}\right) u_{2}+f_{2}(t)\right) d t+\sigma_{2} d W_{2},
\end{aligned}
$$

where $\gamma_{1}, \gamma_{2}$ and $\sigma_{1}, \sigma_{2}$ are damping and white noise coefficients, respectively, that represent the interaction of the model with the unresolved modes [28, 23, 26]; the small parameter $\varepsilon$ measures the ratio between the deterministic time scales of the fast and slow modes, $a_{0}$ is the nonlinearity coefficient, and $f_{1}$ and $f_{2}$ represent the forcing of the slow and fast modes, respectively. The structure of the model is motivated by the studies of geophysical systems that demonstrate that the central feature of the slow-fast interactions are slow vortical mode, represented by $u_{1}$ here, and fast gravity waves, represented by $u_{2}$ here. Moreover, the tropical moist convection as another major dynamical property of the system is represented by the strong fast forcing $f_{2}$. The fast forcing has a direct impact on the modulation of the fast wave amplitude $\left|u_{2}\right|$. Moreover, filtering mixes the fast modes with the slow mode through observations and, therefore, an indirect impact of the fast forcing can also occur on the slow mode.

In our study, we compare the exact NEKF with the linear KF with model error. We show that for small values of observation time and observation noise, the NEKF performs better than the linear KF with model error. However, as the observation time and/or observation variance increases, we obtain a surprising result: the linear KF performs better than the NEKF on the slowly varying amplitude $\left|u_{2}\right|$ of the fast wave. We investigate this phenomenon and study the range of parameters when it arises.

In section 2, we elaborate on the setup of the model and the structure of the fast forcing, in particular. There, we also give the exact solution and exactly solvable statistics by generalizing the exact solutions of the Kubo oscillator [19, 22]. In section 3 , we study filtering strategies for the slow-fast system and present the results of filter performance. There, we also address the issue of the model error due to linearization. We end the paper with the concluding remarks.

\section{Exact solution and exactly solvable statistics}

2.1. The nonlinear test model with fast forcing. As discussed in the introduction, we consider the system of a slow real mode $u_{1}$ interacting with a fast complex mode $u_{2}$. The evolution of this system is given by a system of stochastic differential equations

$$
\begin{aligned}
& d u_{1}=\left(-\gamma_{1} u_{1}+f_{1}(t)\right) d t+\sigma_{1} d W_{1}, \\
& d u_{2}=\left(\left(-\gamma_{2}+i \omega_{0} / \varepsilon+i a_{0} u_{1}\right) u_{2}+f_{2}(t)\right) d t+\sigma_{2} d W_{2} .
\end{aligned}
$$

Here, $\gamma_{1}$ and $\gamma_{2}$ are the damping parameters of the modes and $\sigma_{1}$ and $\sigma_{2}$ represent the strength of the white noise forcing of the corresponding modes. The small parameter 
$\varepsilon$ characterizes the time scale separation between the slow and the fast modes, $\omega_{0}$ is a deterministic frequency of the fast mode in the units of $\varepsilon$, and $a_{0}$ represents the strength of nonlinear interactions between the modes. System (2.1) and (2.2) is considered with the initial values

$$
\begin{aligned}
& u_{1}\left(t_{0}\right)=u_{10}, \\
& u_{2}\left(t_{0}\right)=u_{20},
\end{aligned}
$$

which are independent Gaussian random variables with the known parameters: $\left\langle u_{10}\right\rangle$, $\left\langle u_{20}\right\rangle, \operatorname{Var}\left(u_{10}\right), \operatorname{Var}\left(u_{20}\right), \operatorname{Cov}\left(u_{20}, u_{10}\right), \operatorname{Cov}\left(u_{20}, u_{20}^{*}\right)$. As in [13], we choose an oscillatory forcing of the slow mode $f_{1}(t)=A \sin (\omega t)$. On the other hand, the forcing $f_{2}$ of the fast mode $u_{2}$ is given by a piecewise constant function of time. Moreover, regions where $f_{2} \neq 0$, which represent the bursts of external activity in the fast mode, are alternated with regions where $f_{2}=0$ and the fast mode is not driven by external forcing. Mathematically, we write

$$
\left\{\begin{array}{l}
f_{2}(t)=c_{j} \text { for } t \in\left[t_{2 j}, t_{2 j+1}\right] \\
f_{2}(t)=0 \text { for } t \in\left[t_{2 j+1}, t_{2 j+2}\right]
\end{array}\right.
$$

where $c_{j}$ are generally non-zero constants. We model the starting times $t_{2 j}$ of the activity bursts in $f_{2}$ to be distributed exponentially with the parameter $\lambda$

$$
\operatorname{Prob}\left(t_{2 j+2}-t_{2 j}<t\right)=1-e^{-\lambda t} \text {. }
$$

The time intervals $\left[t_{2 j}, t_{2 j+1}\right]$ where $f_{2} \neq 0$ are chosen to be of the same length $\tau_{d}$. Furthermore, since we want these intervals to be non-overlapping, we choose only those $t_{2 j+2}$ that satisfy $t_{2 j+2}>t_{2 j+1}=t_{2 j}+\tau_{d}$. This restriction makes the distribution of the time gaps between the sequential bursts in $f_{2}$ different from the pure exponential distribution with mean $1 / \lambda$. However, if the length $\tau_{d}$ of each burst is much smaller than $1 / \lambda$, the deviation of the distribution of the $t_{2 j+2}$ from the exponential distribution is not significant and we can still estimate the average length between the bursts as $1 / \lambda$. The size of the amplitude $c_{j}$ of the fast forcing $f_{2}$ is chosen to be an independent random variable uniformly distributed on the interval $(-B, B)$. Note that various values of the parameters $\lambda, \tau_{d}$, and $B$ lead to various regimes of fast forcing, and, therefore, to different dynamics of the slow-fast system. In the bottom panel of figure 2.1, we demonstrate a sample of the fast forcing as a function of time. There, the values of the parameters are: $B=50, \lambda=0.2, \tau_{d}=0.6$. One of the considerations of choosing the parameters of the fast forcing can be the assumption about the magnitude of the waves $u_{1}$ and $u_{2}$ - they can be either of the same order or one can have larger amplitude than the other. Next, we discuss the exact path-wise solution for $u_{1}$ and $u_{2}$.

2.2. Path-wise solution. The system (2.1) and (2.2) can be solved exactly. The solutions, $u_{1}$ and $u_{2}$, obtained in this fashion, provide a signal that will be filtered. Using an integrating factor, we find the slow mode

$$
u_{1}(t)=u_{10} e^{-\gamma_{1}\left(t-t_{0}\right)}+F_{1}\left(t_{0}, t\right)+\sigma_{1} \int_{t_{0}}^{t} e^{-\gamma_{1}(t-s)} d W_{1}(s),
$$

where

$$
F_{1}\left(t_{0}, t\right)=\int_{t_{0}}^{t} f_{1}(s) e^{-\gamma_{1}(t-s)} d s
$$


Now, we solve equation (2.2) to obtain the fast mode

$$
\begin{array}{r}
u_{2}(t)=e^{-\gamma_{2}\left(t-t_{0}\right)} \psi\left(t_{0}, t\right) u_{20}+\int_{t_{0}}^{t} e^{-\gamma_{2}(t-s)} \psi(s, t) f_{2}(s) d s \\
+\sigma_{2} \int_{t_{0}}^{t} e^{-\gamma_{2}(t-s)} \psi(s, t) d W_{2}(s),
\end{array}
$$

where

$$
\begin{aligned}
\psi(s, t) & =e^{i J(s, t)}, \\
J(s, t) & =\int_{s}^{t}\left(\omega_{0} / \varepsilon+a_{0} u_{1}\left(s^{\prime}\right)\right) d s^{\prime}=(t-s) \omega_{0} / \varepsilon+a_{0} \int_{s}^{t} u_{1}\left(s^{\prime}\right) d s^{\prime} \\
& =J_{D}(s, t)+J_{W}(s, t)+b(s, t) u_{10},
\end{aligned}
$$

where the deterministic part is

$$
J_{D}(s, t)=(t-s) \omega_{0} / \varepsilon+a_{0} \int_{s}^{t} F_{1}\left(t_{0}, s^{\prime}\right) d s^{\prime},
$$

the noisy part is

$$
J_{W}(s, t)=\sigma_{1} a_{0} \int_{s}^{t} d s^{\prime} \int_{t_{0}}^{s^{\prime}} e^{\gamma_{1}\left(s^{\prime \prime}-s^{\prime}\right)} d W_{1}\left(s^{\prime \prime}\right),
$$

and the prefactor of $u_{10}$ is

$$
b(s, t)=\frac{a_{0}}{\gamma_{1}}\left(e^{-\gamma_{1}\left(s-t_{0}\right)}-e^{-\gamma_{1}\left(t-t_{0}\right)}\right) .
$$

2.3. Examples of trajectories and choice of parameters. Here, we demonstrate the examples of typical trajectories given by equations (2.7) and (2.9). In order to calibrate the parameters, we use physical intuition which helped us to design the slow-fast system (2.1) and (2.2). The invariant measure for this system without forcing is Gaussian [8] and is given by [13]

$$
p\left(u_{1}, u_{2}\right)=\frac{\sqrt{2 \gamma_{1} \gamma_{2}}}{\pi \sigma_{1} \sigma_{2}} \exp \left(-\frac{\gamma_{1} u_{1}^{2}}{\sigma_{1}^{2}}-\frac{2 \gamma_{2}\left|u_{2}\right|^{2}}{\sigma_{2}^{2}}\right) .
$$

Now, we can choose the parameters for the model in order to control the average energy. For the case of vanishing forcing, the energy equipartition

$$
E_{u_{1}}=E_{R e\left[u_{2}\right]}=E_{I m\left[u_{2}\right]}
$$

yields

$$
\frac{\sigma_{1}^{2}}{\gamma_{1}}=\frac{\sigma_{2}^{2}}{2 \gamma_{2}}
$$

According to the assumption of scale separation, the oscillation time of the slow mode is much longer than the oscillation time of the fast mode. We choose the slow forcing frequency to be $\omega=1$ and the forcing amplitude to be $A=1$. We used the nonlinear parameter $a_{0}=1$. Then, the slow mode has the period of the order 
$2 \pi$. On the other hand, the fast mode has the period of the order $T_{2}=2 \pi \varepsilon / \omega_{0}$. We choose $\omega_{0}=1$ and $\varepsilon=0.1$, then the fast mode has the period of the order $\varepsilon T_{2}$. Next, we choose the typical decorrelation time that is proportional to the inverse of the corresponding damping coefficient. We take $\gamma_{1}=0.09$ and $\gamma_{2}=0.08$ such that $T_{2} \ll 1 / \gamma_{1}$ and $T_{2} \ll 1 / \gamma_{2}$. Suppose that the average energy of the slow mode is $E_{u_{1}}=1$. Then in the energy equipartition case, we have $\sigma_{1}=\sqrt{\gamma_{1}}=0.3$ and $\sigma_{2}=\sqrt{\gamma_{2}}=0.4$. However, the parameters for the fast forcing can affect the average energy as well. We present two situations. In the first case, the fast forcing is chosen so strong that the amplitude of the fast mode is significantly larger than the amplitude of the slow mode. This case is more interesting from the academic point of view since it provides a good test case for the filtering of the strongly forced systems. On the other hand, in the second case, we consider moderate fast forcing, when the amplitude of the fast mode is of the same order as the amplitude of the slow forcing. This case is intended to model the typical realistic situation of the interaction of slow and fast waves.

In figure 2.1, we show the case of strong fast forcing. The parameters of the fast forcing (panel 5 in figure 2.1), are shown there schematically $B=50, \lambda=0.2$, and $\tau_{d}=0.6$. Note the piecewise constant structure of $f_{2}$. Panel 1 of figure 2.1 shows the evolution of the slow mode that was computed via equation (2.7). We note a random structure on the periodic background. Panel 2 of figure 2.1 demonstrates the highly oscillatory behavior of $u_{2}$ computed via equation (2.9) with $a_{0}=1$, i.e., in the nonlinear regime. We note the sharp changes in the amplitude of $u_{2}$ induced by the bursts of activity in $f_{2}$. Moreover, we observe how damping results in a gradual amplitude decrease when $f_{2}$ stays zero until the next burst (e.g., the segment for $t \in[30,50]$ in Panel 2 of figure 2.1). In order to study the effect of nonlinearity, we show the trajectory of $u_{2}$ that is computed with the linear version of equation (2.9), i.e., with $a_{0}=0$ (Panel 3 of figure 2.1). We used the same value of the noise $W_{2}(t)$ for both the nonlinear and linear versions of $u_{2}$. The linear trajectory also reacts by sudden increases of the amplitude of $u_{2}$ when the forcing in the fast mode switches on. However, we note that the nonlinear trajectory has a larger amplitude on average when compared to the linear trajectory. However, there are regions where the linear trajectory has a larger amplitude, such as the region with $t \in[70,90]$ in Panel 3 of figure 2.1. Therefore, we conclude that both linear and nonlinear trajectories have their amplitudes amplified by the fast forcing; however, the dynamics of the amplitudes is different. Panel 4 of figure 2.1 shows the amplitude $\left|u_{2}\right|$ of both linear and nonlinear regimes. Note that filtering of the slowly varying amplitude $\left|u_{2}\right|$ of the fast mode as opposed to the fast mode $u_{2}$ itself will be one of the problems that will be studied below since this skill in capturing the envelope is important for applications. We will find out that under certain conditions, the linear filter with model error actually performs better on the amplitude $\left|u_{2}\right|$ than the exact nonlinear filter. Below, we also study the effects of using a linear filter with model error.

In order to generate the truth signal we use the following procedure. We choose the profile of the fast forcing $f_{2}$ as discussed in section 2.1. We consider any random Gaussian initial data (2.3) and (2.4) and obtain a realization of the trajectory $\left(u_{1}, u_{2}\right)$ using the exact solutions given by equations (2.7) and (2.9). Note that $u_{1}$ can be easily computed since its random part is Gaussian with known statistics. However, $u_{2}$ is not Gaussian and its random part depends on the evolution of $u_{1}$. Therefore, even if we need the true trajectory only at discrete times with a time step $\Delta t$, we still need to compute $u_{1}$ with a much finer resolution with time step $h$. This fine trajectory of $u_{1}$ is then used to compute $u_{2}$. The integrals in equation (2.9) are approximated 

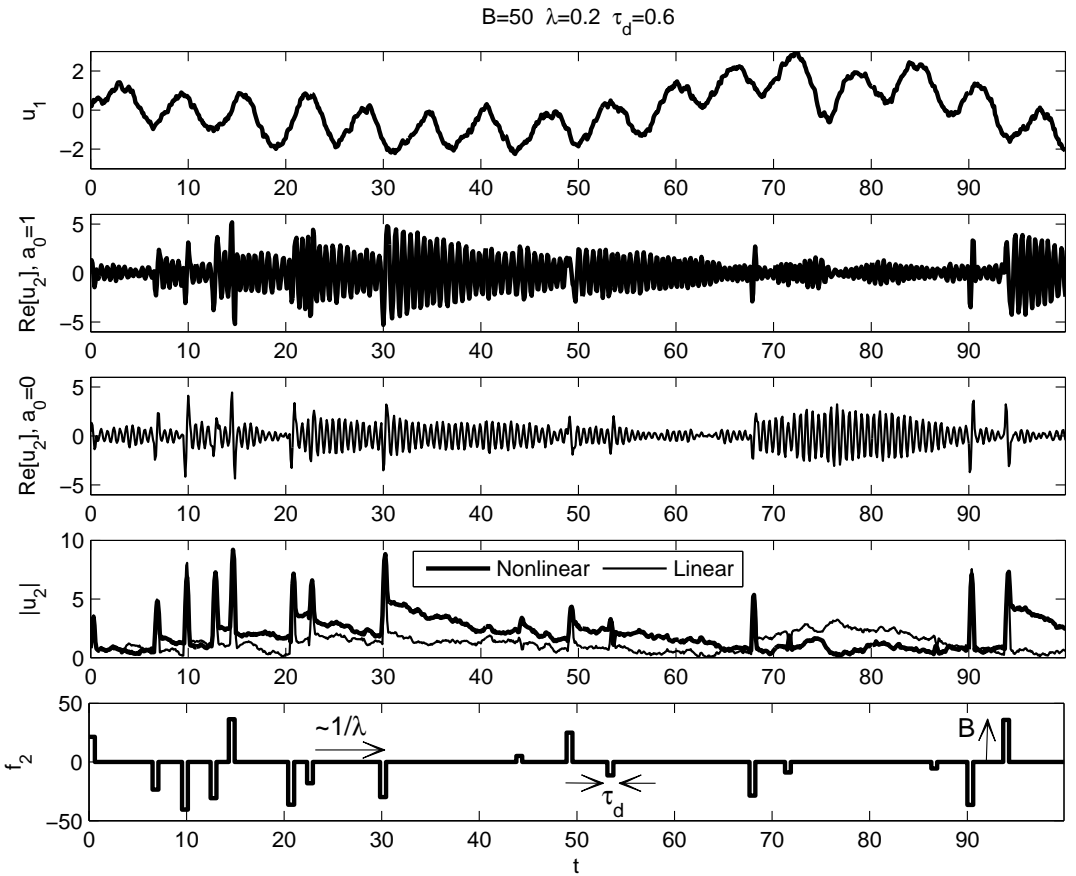

FIG. 2.1. Panel 1: slow mode $u_{1}$; panel 2: real part of nonlinear fast mode $u_{2}$; panel 3: real part of linear fast mode $u_{2}$; panel 4: magnitude of linear and nonlinear $u_{2}$; panel 5: fast forcing $f_{2}$ with schematic notations for parameters $B, \lambda$, and $\tau_{d}$.

numerically using the first order accurate quadrature formula for the deterministic integral

$$
\int_{t_{0}}^{t} g(s) d s \approx h \sum_{j=0}^{N-1} g\left(t_{0}+j h\right),
$$

and the half-order (in the strong sense [12]) accurate formula for the stochastic integral

$$
\int_{t_{0}}^{t} g(s) d W(s) \approx \sum_{j=0}^{N-1} g\left(t_{0}+j h\right) \Delta W_{j}
$$

where $h=\left(t-t_{0}\right) / N$ is a time step in the equidistant partition of the interval $\left[t_{0}, t\right]$ into $N$ subintervals and $\Delta W_{j}$ is independent Gaussian random variables with mean 0 and variance $h$.

2.4. Statistics of $u_{1}$ and $u_{2}$. The slow mode $u_{1}$ is Gaussian with mean and variance given by

$$
\begin{aligned}
\left\langle u_{1}\right\rangle & =\left\langle u_{10}\right\rangle e^{-\gamma_{1}\left(t-t_{0}\right)}+F_{1}\left(t_{0}, t\right), \\
\operatorname{Var}\left(u_{1}\right) & =\operatorname{Var}\left(u_{10}\right) e^{-2 \gamma_{1}\left(t-t_{0}\right)}+\frac{\sigma_{1}^{2}}{2 \gamma_{1}}\left(1-e^{-2 \gamma_{1}\left(t-t_{0}\right)}\right),
\end{aligned}
$$


where we used the following two properties of the Ito stochastic integral: (i) the mean of the Ito stochastic integral is zero and (ii) the Ito isometry formula [12]

$$
\left\langle\left(\int g(t) d W(t)\right)^{2}\right\rangle=\int g^{2}(t) d t
$$

for any deterministic $g(t)$. Next, we obtain the mean of $u_{2}$ by averaging equation $(2.9)$

$$
\left\langle u_{2}\right\rangle=e^{-\gamma_{2}\left(t-t_{0}\right)}\left\langle\psi\left(t_{0}, t\right) u_{20}\right\rangle+\int_{t_{0}}^{t} e^{-\gamma_{2}(t-s)}\langle\psi(s, t)\rangle f_{2}(s) d s .
$$

We start with the second term in the right hand side of equation (2.19). Since $J(s, t)$ is Gaussian, using the properties of the characteristic function of a Gaussian random field we find

$$
\langle\psi(s, t)\rangle=\langle\exp (i J(s, t))\rangle=\exp \left(i\langle J(s, t)\rangle-\frac{1}{2} \operatorname{Var}(J(s, t)),\right.
$$

where

$$
\begin{aligned}
\langle J(s, t)\rangle & =J_{D}(s, t)+b(s, t)\left\langle u_{10}\right\rangle, \\
\operatorname{Var}(J(s, t)) & =\operatorname{Var}\left(J_{W}(s, t)\right)+b^{2}(s, t) \operatorname{Var}\left(u_{10}\right) .
\end{aligned}
$$

Making use of the Ito isometry formula (2.18), we find

$$
\begin{aligned}
& \operatorname{Var}\left(J_{W}(s, t)\right) \\
= & -\frac{\sigma_{1}^{2} a_{0}^{2}}{2 \gamma_{1}^{3}}\left(2+2 \gamma_{1}(s-t)+2 e^{-\gamma_{1}\left(s+t-2 t_{0}\right)}\left(-1-e^{2 \gamma_{1}\left(s-t_{0}\right)}+\cosh \left(\gamma_{1}(s-t)\right)\right)\right) .
\end{aligned}
$$

The integral in the right hand side of equation (2.19) can be approximated numerically via the quadrature formula (2.14). Moreover, using a special piecewise constant structure of $f_{2}$, this integral can be simplified and only computed over the intervals where $f_{2} \neq 0$. We continue with the first term in the right hand side of equation (2.19). Using independence of the noise $W_{1}(t)$ of the initial conditions, we obtain

$$
\left\langle u_{20} \psi\left(t_{0}, t\right)\right\rangle=\psi_{D}\left(t_{0}, t\right)\left\langle\psi_{W}\left(t_{0}, t\right)\right\rangle\left\langle u_{20} \exp \left(i b\left(t_{0}, t\right)\right)\right\rangle,
$$

where

$$
\begin{aligned}
\psi_{D}\left(t_{0}, t\right) & =e^{i J_{D}\left(t_{0}, t\right)} \\
\left\langle\psi_{W}\left(t_{0}, t\right)\right\rangle & =e^{-\frac{1}{2} \operatorname{Var}\left(J_{W}\left(t_{0}, t\right)\right)} \\
\left\langle u_{20} e^{i b\left(t_{0}, t\right) u_{10}}\right\rangle & =\left(\left\langle u_{20}\right\rangle+i \operatorname{Cov}\left(u_{20}, u_{10}\right) b\left(t_{0}, t\right)\right) e^{i b\left(t_{0}, t\right)\left\langle u_{10}\right\rangle-\frac{1}{2} b^{2}\left(t_{0}, t\right) \operatorname{Var}\left(u_{10}\right)} .
\end{aligned}
$$

equation (2.22) is computed via the properties of the characteristic function of a Gaussian random field. The details of this computation are presented in [13]. Next, we compute the variance of $u_{2}$

$$
\operatorname{Var}\left(u_{2}\right)=\left\langle u_{2} u_{2}^{*}\right\rangle-\left|\left\langle u_{2}\right\rangle\right|^{2} .
$$


Since $\left|\left\langle u_{2}\right\rangle\right|^{2}$ can be found using equation (2.19), we only need to find the correlator $\left\langle u_{2} u_{2}^{*}\right\rangle$ to compute $\operatorname{Var}\left(u_{2}\right)$. Using equation (2.9), we find

$$
\begin{aligned}
\left\langle u_{2} u_{2}^{*}\right\rangle= & e^{-2 \gamma_{2}\left(t-t_{0}\right)}\left\langle\left|u_{20}\right|^{2}\right\rangle+\sigma_{2}^{2} \int_{t_{0}}^{t} e^{-2 \gamma_{2}(t-s)} d s \\
& +\int_{t_{0}}^{t} d s \int_{t_{0}}^{t} d r e^{-\gamma_{2}(2 t-s-r)}\left\langle\psi(s, t) \psi^{*}(r, t)\right\rangle f_{2}(s) f_{2}^{*}(r) \\
& +\left(e^{-\gamma_{2}\left(t-t_{0}\right)} \int_{t_{0}}^{t} e^{-\gamma_{2}(t-s)}\left\langle u_{20} \psi\left(t_{0}, t\right) \psi^{*}(s, t)\right\rangle f_{2}^{*}(s) d s+c . c .\right)
\end{aligned}
$$

where we have used the fact that $|\psi|^{2}=1$ and the notation c.c. stands for complex conjugate. Next, using the definition of $\psi(s, t)$, we find that

$$
\psi(s, t) \psi^{*}(r, t)=\psi(s, r)
$$

The integral in the third term of equation (2.24) is real and, therefore, only the real part of $\psi(s, r)$ should be considered. After some simplifications, we find

$$
\begin{aligned}
\left\langle u_{2} u_{2}^{*}\right\rangle= & e^{-2 \gamma_{2}\left(t-t_{0}\right)}\left(\operatorname{Var}\left(u_{20}\right)+\left|\left\langle u_{20}\right\rangle\right|^{2}\right)+\frac{\sigma_{2}^{2}}{2 \gamma_{2}}\left(1-e^{-2 \gamma_{2}\left(t-t_{0}\right)}\right) \\
& +\int_{t_{0}}^{t} d s \int_{t_{0}}^{t} d r f_{2}(s) f_{2}(r) e^{-\gamma_{2}(2 t-s-r)} \operatorname{Re}[\langle\psi(s, r)\rangle] \\
& +\left(e^{-\gamma_{2}\left(t-t_{0}\right)} \int_{t_{0}}^{t} f_{2}(s) e^{-\gamma_{2}(t-s)}\left\langle u_{20} \psi\left(t_{0}, s\right)\right\rangle d s+c . c .\right)
\end{aligned}
$$

where we used equations (2.20) and (2.21) to find the integrands in equation (2.26). Here, again we use the piecewise constant structure of $f_{2}$ and the quadrature formula (2.14) to approximate the integrals. In a similar manner, we find the crosscovariances of $\operatorname{Cov}\left(u_{2}, u_{1}\right)$ and $\operatorname{Cov}\left(u_{2}, u_{2}^{*}\right)$. Since these computations are tedious, we placed them in the Appendix.

2.5. Analytical formulas and Monte Carlo averaging. In this section, we demonstrate how the results of the analytical formulas for the statistics of $u_{1}$ and $u_{2}$ match the results of Monte Carlo simulation. We use the ensemble of $M=10^{4}$ members in Monte Carlo averaging. We choose one arbitrary profile of the strong forcing $f_{2}$ with the parameters $\lambda=1.3, \tau_{d}=0.6$, and $B=50$. In figure 2.2 , we demonstrate the time evolution of the various first and second order statistics of $u_{1}$ and $u_{2}$ together with the profile of the fast forcing $f_{2}$. There, the fast forcing $f_{2}$ has a very abrupt behavior and the statistics reflect to the bursts of activity in $f_{2}$ (regions where $\left.f_{2} \neq 0\right)$ by strong amplitude modulations. We note excellent agreement between the analytical formulas and Monte Carlo averaging.

2.6. Nongaussianity of $u_{2}$. One of the main properties of the test model (2.1) and (2.2) is nongaussianity of the fast mode $u_{2}$. Here, we show how the statistics of $u_{2}$ deviate from the corresponding Gaussian values. Both nonlinear structure $\left(a_{0} \neq 0\right)$ and fast forcing $\left(f_{2} \neq 0\right)$ make it natural to expect nongaussian statistics of the fast mode $u_{2}$. As we discussed earlier, the invariant measure of our system is Gaussian. However, the continuously applied fast forcing $f_{2}$ prevents the system from converging 

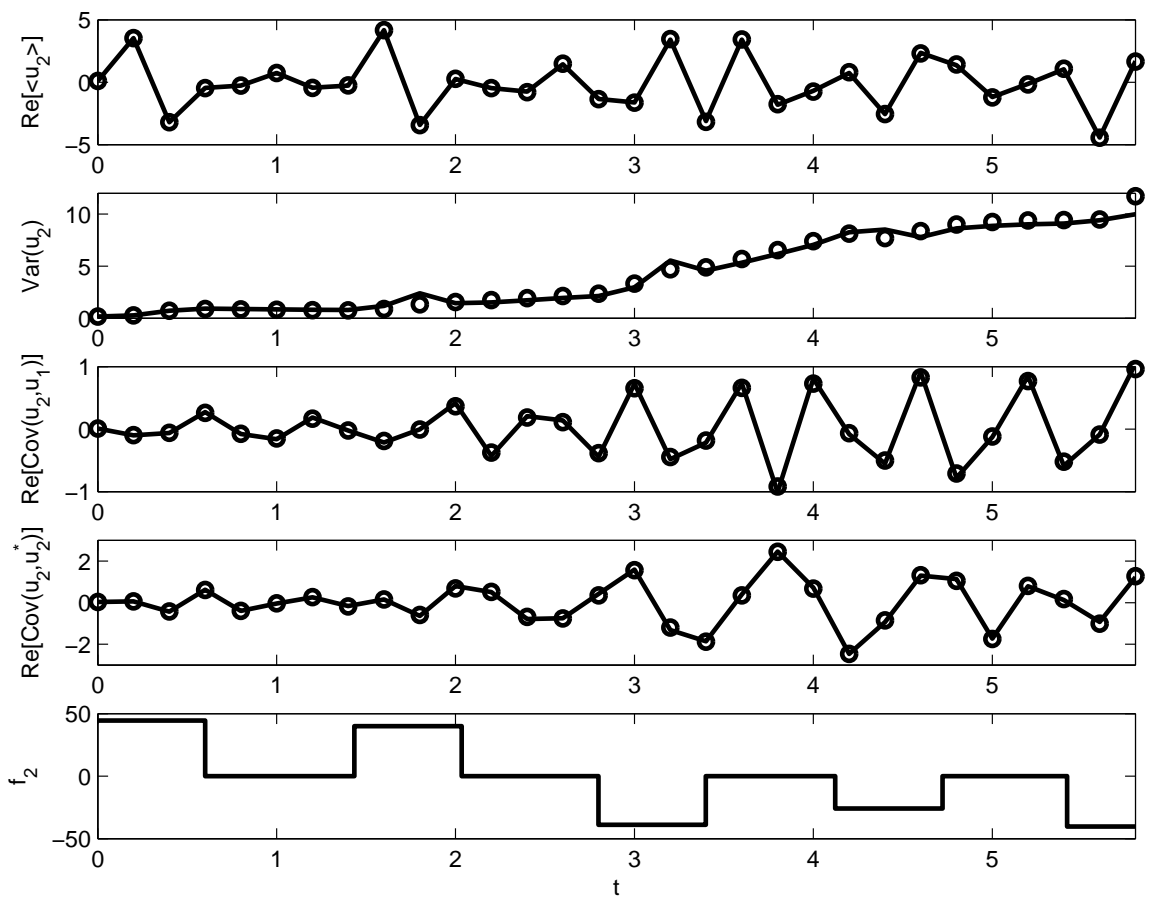

FIG. 2.2. The solid line represents the analytical values of the statistics and circles correspond to the Monte Carlo averaging. First panel: mean $\left\langle u_{2}\right\rangle$; second panel: variance Var $\left(u_{2}\right)$; third panel: covariance $\operatorname{Cov}\left(u_{2}, u_{1}\right)$; fourth panel: covariance $\operatorname{Cov}\left(u_{2}, u_{2}^{*}\right)$; fifth panel: fast forcing $f_{2}$. Note that only real parts are shown for the mean and covariance.

to the Gaussian state. In order to detect the deviation from Gaussian statistics, we measure skewness of the fast mode. For a random variable $\xi$, the skewness is

$$
\text { skewness }(\xi)=\frac{\left\langle(\xi-\langle\xi\rangle)^{3}\right\rangle}{\operatorname{Var}(\xi)^{3 / 2}} .
$$

For Gaussian $\xi$, skewness $(\xi)=0$. Earlier in [13], it was shown numerically that the system $(2.1)$ and $(2.2)$ without fast forcing $\left(f_{2} \equiv 0\right)$ has the skewness and kurtosis [12] of the fast mode $u_{2}$ converge to their Gaussian values ( 0 and 3, respectively) asymptotically as time evolves. In figure 2.3 , we show evolution of the skewness of $\operatorname{Re}\left[u_{2}\right]$ for both $f_{2} \neq 0$ and $f_{2} \equiv 0$. Note that in the case of a piecewise constant $f_{2}$, the deviation of the skewness from zero is very strong and does not vanish as time progresses. On the other hand, for $f_{2} \equiv 0$ the skewness is much weaker and approaches zero after the decorrelation time.

\section{Filtering}

In this section, we first briefly discuss a general theory of the Nonlinear Extended Kalman filter (NEKF); then, we demonstrate the performance of the NEKF on the nonlinear test model with fast forcing. 



FIG. 2.3. Upper panel: the skewness as a function of time; lower panel: the forcing $f_{2}$. The solid line corresponds to the case of a piecewise constant $f_{2}$ which is not identically equal to zero and the dashed line corresponds to the case $f_{2} \equiv 0$.

3.1. Nonlinear Extended Kalman filter. The linear Kalman filter $[1,5]$ is used when the best possible approximation to the truth signal is needed. The algorithm for finding this optimal approximation incorporates the assumed (linear) dynamics with the observations of the signal and uses least squares minimization of the error. Both dynamics and observations are characterized by deterministic and Gaussian stochastic components. Because of the Gaussian statistics, the dynamics of the ensemble can be fully described by the propagation of the mean and covariance. The NEKF is a generalization of the linear KF for nonlinear systems. Similar to the linear KF, the NEKF only requires the model for propagation of the mean and covariance. However, due to nonlinear dynamics, the Gaussian ensemble of initial data may not (and generally does not) stay Gaussian as time progresses, which makes the NEKF only suboptimal.

Let us introduce the extended Kalman filter algorithm for the test model (2.1) and (2.2). Suppose that at time $t_{m}=m \Delta t$, where $m \geq 0$ is an observation time step index and $\Delta t$ is the observation time step, the truth signal is denoted as $\mathbf{u}_{m}$, which is a realization of $\left(u_{1}, u_{2}\right)$ computed via equations $(2.7)$ and $(2.9)$. We assume that $\mathbf{u}_{m}$ is unknown and we are instead given an observation of the truth signal, which is a linear transformation of $\mathbf{u}_{m}$ mixed with some Gaussian noise

$$
\mathbf{v}_{m}=G \mathbf{u}_{m}+\sigma_{m}^{0}
$$

where $G$ is a rectangular matrix of the size $q \times 3$ with the number of observations $q=\{1,2,3\}, \mathbf{u}=(x, y, z)^{T} \equiv\left(u_{1}, \operatorname{Re}\left[u_{2}\right], \operatorname{Im}\left[u_{2}\right]\right)^{T}$ and $\sigma_{m}^{0}$ is the observation noise. The 
observation noise is assumed to be unbiased (mean-zero) with covariance matrix $R^{0}$ of the size $q \times q$. The goal of filtering is to find the filtered signal $\mathbf{u}^{f}$, which is as close as possible to the original truth signal $\mathbf{u}$. The information that can be used in filtering is limited to

- the model for dynamical evolution of $\mathbf{u}_{m}$,

- the matrix $G$, and

- the mean and covariance of the Gaussian noise $\sigma_{m}^{0}$.

The Kalman filter $[1,5]$ consists of two steps: (i) forecasting using the dynamics and (ii) correcting using observations. Denote the mean and covariance of the filtered signal at time $t_{m}$ as $\langle\mathbf{u}\rangle_{m \mid m}$ and $\Gamma_{m \mid m}$, respectively. Then the forecast step gives us the following so called prior values of the mean and covariance at the next time step $t_{m+1}$ :

$$
\begin{aligned}
\langle\mathbf{u}\rangle_{m \mid m} & \rightarrow\langle\mathbf{u}\rangle_{m+1 \mid m}, \\
\Gamma_{m \mid m} & \rightarrow \Gamma_{m+1 \mid m} .
\end{aligned}
$$

Note that $\langle\mathbf{u}\rangle_{m+1 \mid m}$ and $\Gamma_{m+1 \mid m}$ depend solely on the prior information up to time $t_{m}$. The posterior values of mean and covariance are obtained by utilizing the observations $\mathbf{v}_{m+1}$ at time $t_{m+1}$ via the least squares correction method

$$
\begin{aligned}
\langle\mathbf{u}\rangle_{m+1 \mid m+1} & =\langle\mathbf{u}\rangle_{m+1 \mid m}+K_{m+1}\left(\langle\mathbf{v}\rangle_{m+1}-G\langle\mathbf{u}\rangle_{m+1 \mid m}\right), \\
\Gamma_{m+1 \mid m+1} & =\left(I_{3}-K_{m+1} G\right) \Gamma_{m+1 \mid m}, \\
K_{m+1} & =\Gamma_{m+1 \mid m} G^{T}\left(G \Gamma_{m+1 \mid m} G^{T}+R^{0}\right)^{-1},
\end{aligned}
$$

where $K_{m+1}$ is a Kalman gain matrix of the size $3 \times q$ and $I_{3}$ is the identity $3 \times 3$ matrix. For the linear forecast model, the posterior distribution is the Gaussian distribution with the mean and covariance given in equation (3.3). However, in the nonlinear dynamics, the prior and posterior distributions are not Gaussian. However, the posterior distribution becomes the initial distribution for the next assimilation cycle, which we again assume to be Gaussian. We note that Kalman gain $K_{m}$ tells us how much weight the filter puts on the observations vs prior forecast.

3.2. Observations. In a typical slow-fast system, such as the shallow water equations, observation of pressure, temperature, and velocity automatically mixes the fast and slow components and can corrupt the filtering of the slow component. Here, we consider exactly the same types of observations that were used by the authors in [13] for the slow-fast system without forcing. In Table 3.1, we present these prototype observations. According to equation (3.1), observations are defined by the transformation matrix $G$ and covariance matrix $R^{0}$. We will consider three different types of observations with the corresponding matrices $G$ and $R^{0}$. Here, we assumed that the components of the observation noise $\sigma_{m}^{0}$ are independent mean-zero Gaussian with variances given by $R^{0}$. Note that observations of types 1 and 2 provide only partial information about the truth signal. Moreover, even initially independent slow and fast modes become correlated through the nonlinearity in equation (2.2) and through mixed observation and equation (3.3). On the other hand the more idealistic observations of type 3 are studied here to provide a benchmark for observations of types 1 and 2, which are more practical.

3.3. Linear filter with model error. Now, suppose that the prior forecast $\mathbf{u}_{m+1 \mid m}$ is made using the linearized version of the analytical equations that we obtained in section 2 . We set $a_{0}=0$ in equation (2.2) and thus we introduce the model 


\begin{tabular}{|c|c|c|}
\hline observation type & $G$ & $R^{0}$ \\
\hline 1 & $\left(1 \frac{1}{\sqrt{2}} \frac{1}{\sqrt{2}}\right)$ & $2 r^{0}$ \\
\hline 2 & $\left(\begin{array}{cc}1 \frac{1}{\sqrt{2}} & \frac{1}{\sqrt{2}} \\
1 & \frac{1}{\sqrt{2}}-\frac{1}{\sqrt{2}}\end{array}\right)$ & $\left(\begin{array}{cc}2 r^{0} & 0 \\
0 & 2 r^{0}\end{array}\right)$ \\
\hline 3 & $\left(\begin{array}{ccc}1 & \frac{1}{\sqrt{2}} & \frac{1}{\sqrt{2}} \\
1 & \frac{1}{\sqrt{2}} & -\frac{1}{\sqrt{2}} \\
1 & 0 & 0\end{array}\right)$ & $\left(\begin{array}{ccc}2 r^{0} & 0 & 0 \\
0 & 2 r^{0} & 0 \\
0 & 0 & r^{0}\end{array}\right)$ \\
\hline
\end{tabular}

TABLE 3.1. Three type of observations given by equation (3.1) via the transformation matrix $G$ and covariance matrix $R^{0}$.

error. As was studied in [13] for the slow-fast system without fast forcing, in the long run the correlation between the slow wave $u_{1}$ and fast wave $u_{2}$ vanishes and so does the effect of nonlinear coupling through $a_{0}$. On the other hand, with fast forcing, the nongaussianity is very strong and the model error due to linearization can be larger than for the case of no fast forcing. Below, we will study the performance of the filter with model error. In particular, we will be interested how the linear filter performs on the slow mode and the slowly varying amplitude $\left|u_{2}\right|$ of the fast mode since often only the slow dynamics of the system is important.

The advantage of using a linear model as an approximation of the true dynamics is also for practical application. In real physical problems, the true dynamics of the model is often unknown and ensemble approximations to the Kalman filter are very expensive for a large dimensional system. Thus, the performance of the linear filter in the nonlinear test model for the slow-fast system is interesting for several reasons $[16,17]$. Note that the truth signal is always produced via the nonlinear version of equations (2.1) and (2.2) with $a_{0} \neq 0$. Therefore, if we use the linear approximation to the original system we may not obtain the optimal filtered signal due to model error. Below, we will compare the error in filtering the test problem using the NEKF and the linear $\mathrm{KF}$ with model error.

In our test model, linearization only affects the fast mode $u_{2}$. Substituting $a_{0}=0$ into equation (2.19) yields the following linear equation

$$
\left\langle u_{2}\right\rangle=e^{\left(-\gamma_{2}+i \omega_{0} / \varepsilon\right) \Delta t}\left\langle u_{20}\right\rangle+\int_{t_{0}}^{t} e^{\left(-\gamma_{2}+i \omega_{0} / \varepsilon\right)(t-s)} f_{2}(s) d s .
$$

Therefore, the forecast is made according to

$$
\langle\mathbf{u}\rangle_{m+1 \mid m}=B\langle\mathbf{u}\rangle_{m \mid m}+C,
$$

where

$$
B=\left(\begin{array}{ccc}
e^{-\gamma_{1} \Delta t} & 0 & 0 \\
0 & e^{-\gamma_{2} \Delta t} \cos (\alpha) & -e^{-\gamma_{2} \Delta t} \sin (\alpha) \\
0 & e^{-\gamma_{2} \Delta t} \sin (\alpha) & e^{-\gamma_{2} \Delta t} \cos (\alpha)
\end{array}\right) \text {, with } \alpha=\Delta t \omega_{0} / \varepsilon
$$

and

$$
C=\left(\begin{array}{c}
F_{1}\left(t_{0}, t\right) \\
F_{2}\left(t_{0}, t\right) \\
0
\end{array}\right)
$$


where $F_{1}\left(t_{0}, t\right)$ is given by equation $(2.8)$ and

$$
F_{2}\left(t_{0}, t\right)=\int_{t_{0}}^{t} e^{\left(-\gamma_{2}+i \omega_{0} / \varepsilon\right)(t-s)} f_{2}(s) d s .
$$

Equation (3.5) is used as a prior forecast for the mean. Similarly by substituting $a_{0}=0$ into the expressions for $\operatorname{Var}\left(u_{2}\right)$ (equation $(2.23)$ ) and $\operatorname{Cov}\left(u_{2}, u_{1}\right)$ and $\operatorname{Cov}\left(u_{2}, u_{2}^{*}\right)$ (see Appendix), we obtain the prior covariance of the linearized model.

One of the important properties of the Kalman filter is observability, which shows how trustworthy the observations are $[1,5]$. The analysis of observability of the linearized system (2.7) and (2.9) with $a_{0}=0$ is the same as it was in [13] for the case of no fast forcing. There, in [13], it was shown that with observations of types 1 and 2 , the linearized system looses observability if and only if

$$
\Delta t=2 \pi l \varepsilon / \omega_{0},
$$

for some integer $l$. Practically, the slow fast system can still lack observability even for the case of full observations of type 3 when the observability matrix has full rank but is close to being singular. In the nonlinear case, we also might expect deteriorating filter performance around the values of $\Delta t$ described by equation (3.8).

3.4. Filter performance: individual trajectories. Let us turn to the study of the NEKF performance on the test model (2.1) and (2.2). We choose a realization of the truth signal computed via equations (2.7) and (2.9) as discussed in section 2.3. Then, we apply the NEKF to the given truth signal and measure the difference between the truth signal $\mathbf{u}_{m}$ and the posterior mean $\langle\mathbf{u}\rangle_{m \mid m}$. The filter skill is defined by the proximity of the posterior signal to the truth signal. We use the root mean square error (RMSE) to measure the filter skill

$$
\operatorname{RMSE}(\mathbf{z}-\mathbf{w})=\sqrt{\frac{1}{N} \sum_{j=1}^{N}\left|z_{j}-w_{j}\right|^{2}},
$$

where $\mathbf{z}$ and $\mathbf{w}$ are the complex vectors to be compared and $N$ is the length of each vector. The ratio of the RMSE and the typical magnitude of the signal gives the normalized percentage error.

In figure 3.1, we show the truth signal with the corresponding fast forcing. Note that we also demonstrate the evolution of the amplitude $\left|u_{2}\right|$ of the fast wave. The amplitude $B=15$ of the fast forcing $f_{2}$ was chosen to keep the amplitudes of both slow and fast modes of the same order of size, which is a realistic situation in nature. We compare the filter performance for the case of the NEKF with the mean and covariance computed via the nonlinear equation (see section 2) with $a_{0}=1$ and the linear KF with model error, where mean and covariance are computed with linear versions of the same equations with $a_{0}=0$. We are interested in particular to see how both of these filtering strategies deal with the sudden changes in the amplitude of the fast mode due to the fast forcing. Therefore, we will restrict our attention only to a short segment of the trajectory with $t \in[55,65]$ from figure 3.1 that includes the spike of the fast forcing. We choose two values of the observation time: $\Delta t=0.1$ and $\Delta t=1.0$. The first one is considerably smaller than the typical oscillation period $T_{2}=2 \pi \varepsilon / \omega_{0}$ of the fast mode and the other one is larger than $T_{2}$. We also choose three values of the observation variance: $r^{0}=0.1, r^{0}=1.0$, and $r^{0}=10.0$, which are smaller than, 

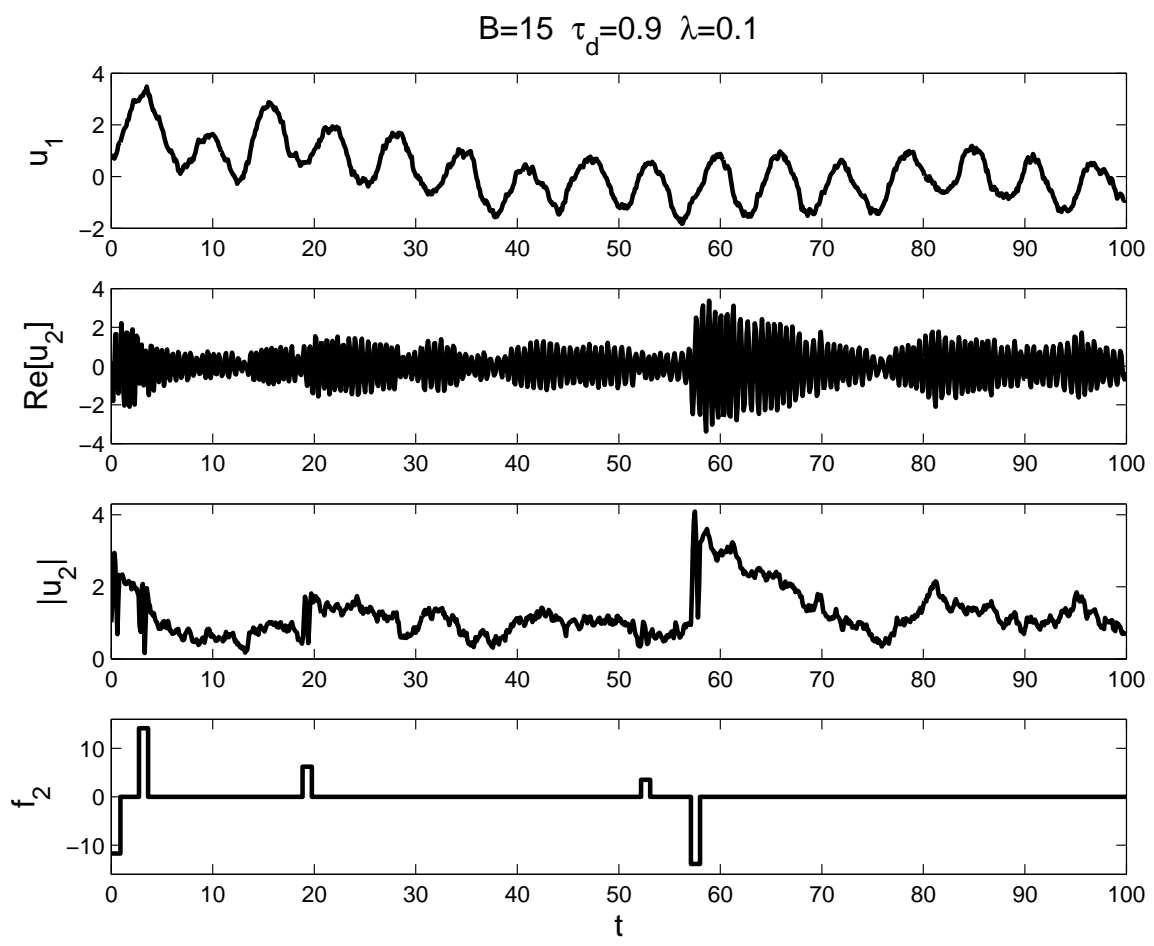

FIG. 3.1. Truth signal $\left(u_{1}, u_{2}\right)$, amplitude $\left|u_{2}\right|$, and fast forcing $f_{2}$ with parameters $B=15$, $\lambda=0.1, \tau_{d}=0.9$.

\begin{tabular}{|c|c|c|}
\hline$r^{0} \backslash \Delta t$ & 0.1 & 1.0 \\
\hline 0.1 & $0.14(0.16)$ & $0.33(0.33)$ \\
\hline 1.0 & $0.22(0.22)$ & $0.35(0.36)$ \\
\hline 10.0 & $0.34(0.34)$ & $0.49(0.50)$ \\
\hline
\end{tabular}

TABLE 3.2. RMSE of the slow mode $u_{1}$ of the exact NEKF and the linear Kalman filter with model error (in parenthesis), observations of type 1. The truth signal is shown in figure 3.1 and the segments of corresponding filtered signals are shown in figure 3.2

of the order of, and larger than the typical size of the truth signal. Moreover, we use observations of type 1 here, because they represent the most interesting practical example of mixed observations. In figure 3.2, we demonstrate the truth signal for the slow mode $u_{1}$ together with the two filtered signals: the NEKF with exact mean and covariance and the linear KF with model error. In Table 3.2, we give the RMSE for $u_{1}$ for the whole trajectory shown in figure 3.1. We note that the filter skill for the slow mode depends on the observation time $\Delta t$ and observation variance $r^{0}-$ the filter skill drops if either of these quantities increases. However, the slow mode is filtered as well with the NEKF as with the linear KF with model error.

Next, we look at the fast mode, which is shown in figure 3.3 and in the corresponding Table 3.3 with RMSE. Here, we again note that the filter becomes less skillful if either the observation time $\Delta t$ or the observation variance $r^{0}$ increase. Furthermore, 

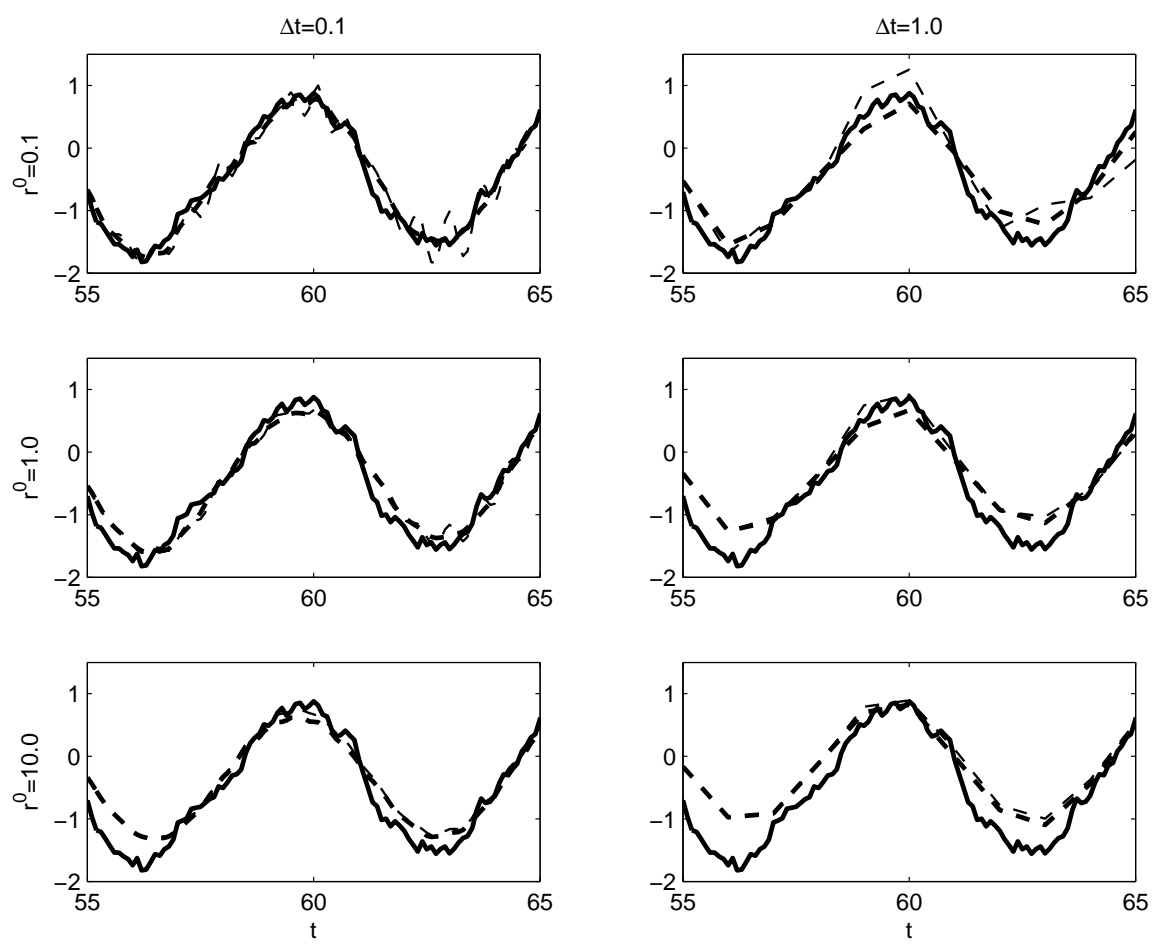

FIG. 3.2. Slow mode $u_{1}$ : truth signal (solid line), the exact NEKF (thick dashed line) and the linear Kalman filter (thin dashed line). The corresponding RMSE for the whole trajectory from figure 3.1 are shown in Table 3.2

\begin{tabular}{|c|c|c|}
\hline$r^{0} \backslash \Delta t$ & 0.1 & 1.0 \\
\hline 0.1 & $0.24(0.61)$ & $0.62(1.10)$ \\
\hline 1.0 & $0.44(0.97)$ & $0.98(1.30)$ \\
\hline 10.0 & $1.05(1.28)$ & $1.25(1.53)$ \\
\hline
\end{tabular}

TABLE 3.3. RMSE of the fast mode $u_{2}$ of the exact NEKF and the linear Kalman filter with model error (in parenthesis), observations of type 1. The truth signal is shown in figure 3.1 and the segments of corresponding filtered signals are shown in figure 3.3

the skill for the fast mode of the linear KF deteriorates much more than of the NEKF. This is naturally expected since the linearization affects the coupling of the fast mode with the slow mode.

However, a surprising result appears when we compare the two filtering strategies for the amplitude $\left|u_{2}\right|$ of the fast mode. In figure 3.4, we demonstrate the true and filtered signals for $\left|u_{2}\right|$. We note that the linear KF gives a much better approximation of the truth for larger $\Delta t$ and $r^{0}$. Table 3.4 confirms this result - the RMSE is significantly smaller for the linear KF than for the NEKF. Therefore, not only is the linear KF easier to compute but it also provides a better approximation of the truth signals when only the envelope of the fast wave is an object of study. This result justifies the use of the cheaper linear filters and demonstrates their effectiveness for the 

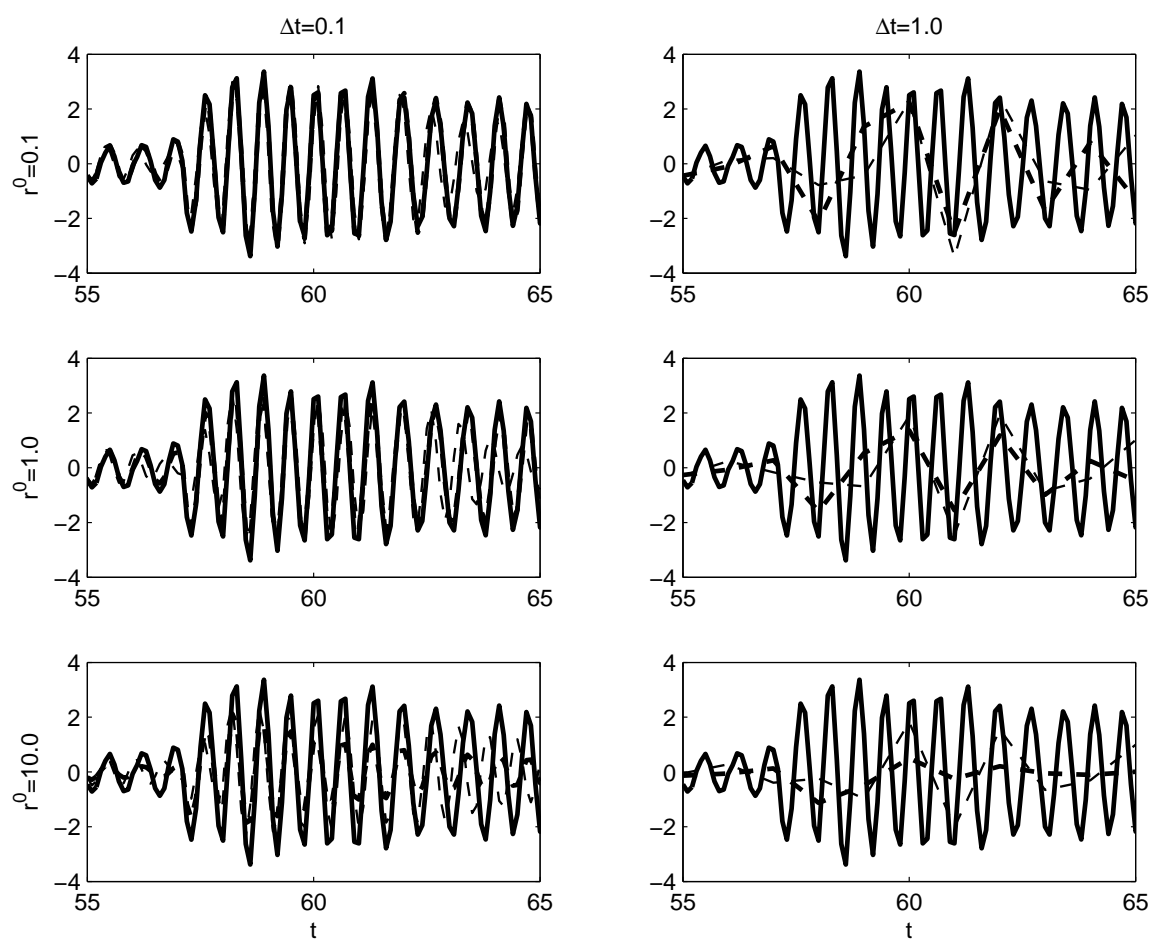

FIG. 3.3. Fast mode $u_{2}$ : truth signal (solid line), the exact NEKF (thick dashed line) and the linear Kalman filter (thin dashed line). The corresponding RMSE for the whole trajectory from figure 3.1 are shown in Table 3.3

\begin{tabular}{|c|c|c|}
\hline$r^{0} \backslash \Delta t$ & 0.1 & 1.0 \\
\hline 0.1 & $0.17(0.28)$ & $0.44(0.52)$ \\
\hline 1.0 & $0.36(0.58)$ & $0.91(\mathbf{0 . 7 7})$ \\
\hline 10.0 & $1.03(\mathbf{0 . 8 2})$ & $1.20(\mathbf{0 . 9 3})$ \\
\hline
\end{tabular}

TABLE 3.4. RMSE of the amplitude $\left|u_{2}\right|$ of the fast mode of the exact NEKF and the linear Kalman filter with model error (in parenthesis), observations of type 1. Truth signal is shown in figure 3.1 and segments of corresponding filtered signals are shown in figure 3.4. Numbers in bold indicate the situations when the linear Kalman filter performs better than the exact NEKF

slow-fast systems with fast forcing. Below, we will study this phenomenon in greater detail by examining the filter performance as a function of $\Delta t, r^{0}$, and observation type.

3.5. Filter performance: dependence on observation type, observation time, and observation variance. In this section, we study the performance of both the NEKF and linear KF for various observation types, observation times $\Delta t$, and observation variance $r^{0}$. We test the statistics of the filter error on two long trajectories. The first one has moderate fast forcing with $B=15$ and, as a result, the magnitudes of both slow and fast modes are of the same order: $\left|u_{1}\right| \sim\left|u_{2}\right| \sim 4$. The second trajectory has strong fast forcing with $B=50$, which results in a stronger 

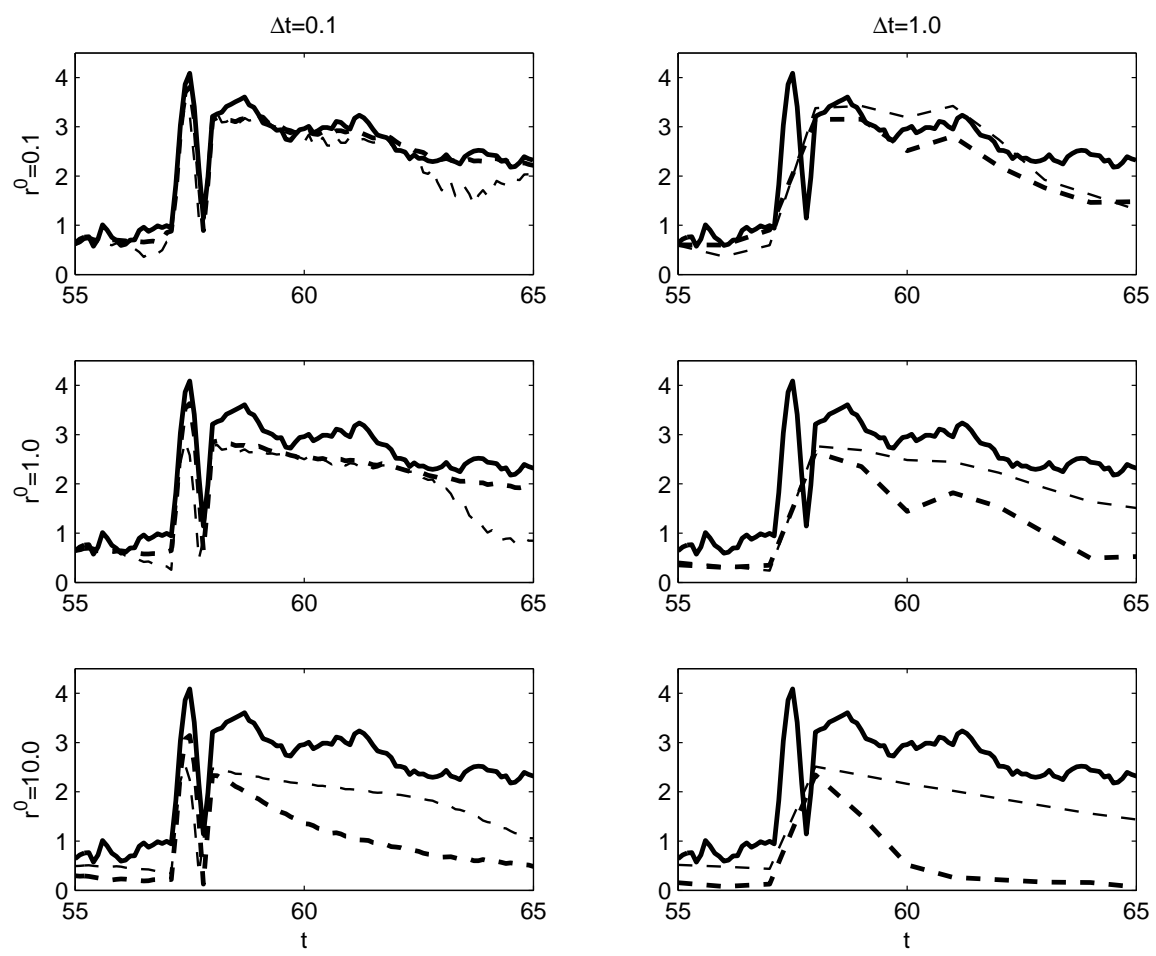

FIG. 3.4. Amplitude $\left|u_{2}\right|$ of the fast mode: truth signal (solid line), the exact NEKF (thick dashed line) and the linear Kalman filter (thin dashed line). The corresponding RMSE for the whole trajectory from figure 3.1 are shown in Table 3.4

fast mode. In this case, we have $\left|u_{1}\right| \sim 4$ and $\left|u_{2}\right| \sim 12$. The second situation is more interesting from the academic point of view since it allows us to test the filtering strategies for a more extreme case. In both situations, the frequency parameter of forcing events and the duration of each event are chosen to be the same, i.e., $\lambda=0.1$ and $\tau_{d}=0.9$, respectively. The frequency parameter is chosen such that the system is allowed to relax in average after each forcing event because the average relaxation time is of the order of $1 / \gamma \sim 10$ as is the average time $\sim 1 / \lambda=10$ between the fast forcing bursts. The duration of each forcing event is chosen to be larger than the typical fast oscillation time $T_{2}=0.6$ but smaller than the time between these events. Both trajectories were computed for the time span $[0, T]$, where $T=5000$.

In figure 3.5, we demonstrate the dependence of the filter performance on the observation time $\Delta t$. There, we use the same moderate observation variance $r^{0}=0.3$. We note that the RMSE depicted in figure 3.5 has peaks which correspond to the lack of observability and which were predicted by linear observability analysis in equation (3.8) (see [13]). We also note that observations of type 3 give the smallest error and observations of type 1 give the largest error, which is an expected result because observations of type 3 carry the most information and observations of type 1 carry the least information among all three types of observations (see Table 3.1). However, there is not much difference between the observations of types 2 and 3 when the RMSE of the fast mode is studied. This is explained by the fact that the observations 



FIG. 3.5. RMSE as a function of observation time $\Delta t$. The left column corresponds to the moderate fast forcing with $B=15$ and the right column corresponds to the strong fast forcing with $B=50$. The first row shows the RMSE of the slow mode, the second row shows the RMSE of the fast mode, and the third row shows the RMSE of the amplitude of the fast mode. Thick lines correspond to the exact NEKF and thin lines correspond to the linear Kalman filter. Solid lines correspond to observations of type 1, dashed lines correspond to observation of type 2, and dashed-dotted lines correspond to observations of type 3. The observation variance was $r^{0}=0.3$.

of type 3 provide additional information mostly about the slow mode, which only slightly reflects the filtering of the fast mode. The NEKF appears to be much more efficient than the linear KF with model error when the either the slow mode $u_{1}$ or the fast mode $u_{2}$ are filtered. However, when the amplitude of the fast mode $\left|u_{2}\right|$ becomes an object of study, for larger $\Delta t$, the RMSE of the linear filter with model error is not larger than the RMSE of the exact NEKF (the thick and thin solid lines cross on the left lower panel in figure 3.5). This phenomenon was already observed above for the path-wise filtering (see figure 3.4). Next, we compare the filter performance for the two trajectories. We note that both the NEKF and the linear KF have larger errors when applied to the trajectory with the strong fast forcing compared with the trajectory with the moderate fast forcing. However, we should keep in mind that the RMSE have to be normalized by the typical magnitudes of the signals, which are larger for the case of strong fast forcing than for the case of moderate fast forcing. We also would like to point out that the NEKF works well and provides stable filtering even for the case of strong fast forcing and hence for strong nongaussianity.

In order to compare the NEKF and the linear KF, we test both of them on the amplitude $\left|u_{2}\right|$ of the trajectory with moderate fast forcing. In figure 3.6, we 



FIG. 3.6. RMSE of filtering of $\left|u_{2}\right|$ as a function of observation time $\Delta t$. The columns correspond to observation types (shown on the top). The rows correspond to various noise variances (shown on the left). The thick line shows the RMSE of the exact NEKF and the thin line shows the RMSE of the linear Kalman filter. The trajectory with the moderate forcing with $B=15$ was filtered.

compare the filter performance for all three types of observations and various values of observation variance. We note that for the chosen range of $\Delta t \in[0,2]$, the RMSE of the linear Kalman filter is practically independent of $\Delta t, r^{0}$, or the observation variance, whereas the RMSE of the exact NEKF is growing if either $\Delta t$ or $r^{0}$ increases or the number of observations decreases. We observe that for $\Delta t$ larger than certain values (dependent on $r^{0}$ and observation type), the linear $\mathrm{KF}$ is more skillful in filtering of $\left|u_{2}\right|$ than the NEKF. We have chosen three different sets of the observation parameters and, in figure 3.7, we compare filtering of $\left|u_{2}\right|$ for these sets of parameters. In figure 3.7, the first panel corresponds to figure 3.6, second row and first column, with observation type $1, r^{0}=0.9$, and $\Delta t=1.6$. We note that the signal obtained with the linear KF follows the truth signal better than the signal obtained with the NEKF. The second panel of figure 3.7 corresponds to figure 3.6, third row and first column, with observation type $1, r^{0}=2.1$, and $\Delta t=1.6$. Here, again the linear filter performs better than the NEKF. Finally, the third panel of figure 3.7 corresponds to figure 3.6 , third row and third column, with observation type $3, r^{0}=2.1$, and $\Delta t=2.0$. And again we have the prevailing of the linear filter over the NEKF.

We have studied how the performance of both linear and nonlinear filters depends on the observation time $\Delta t$. We have found that the linear KF with model error has higher skill in filtering the slow amplitude $\left|u_{2}\right|$ when compared with the NEKF for 

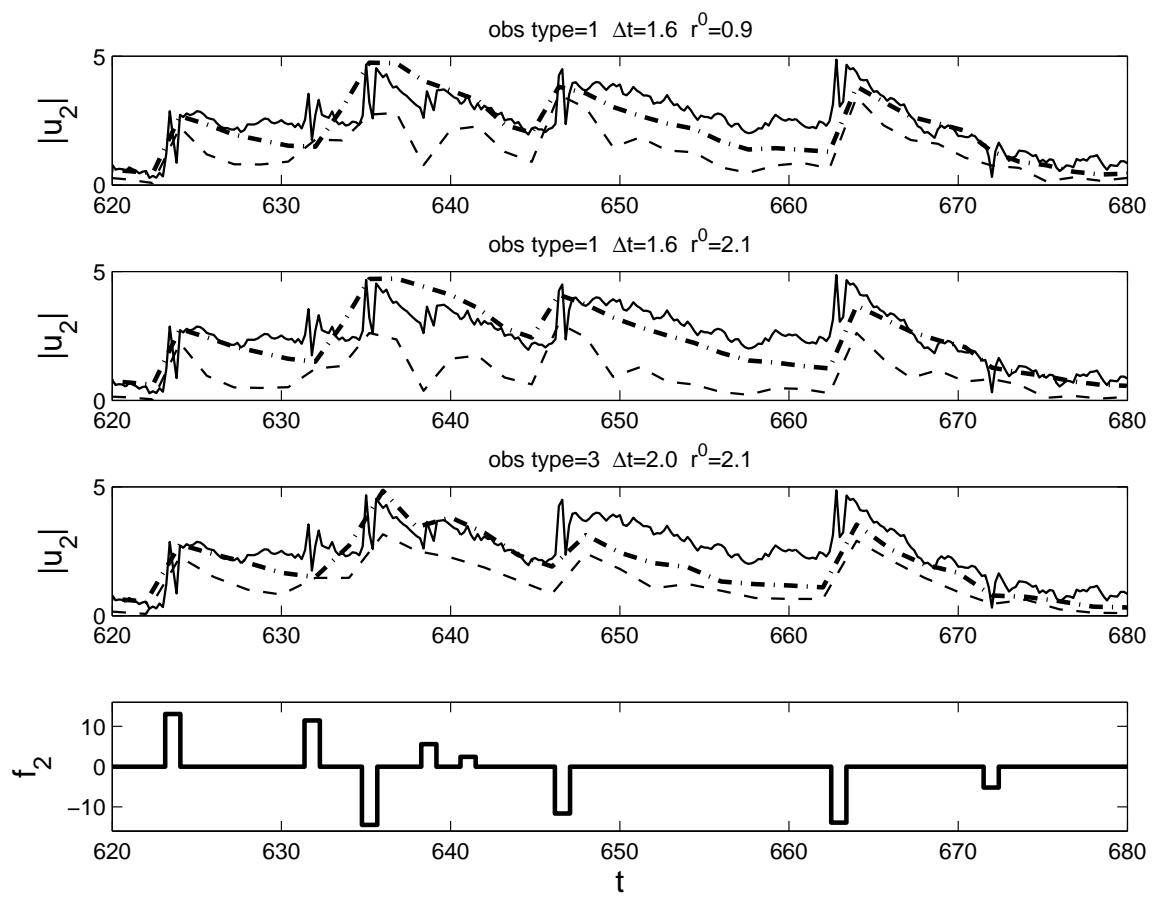

FIG. 3.7. Comparison of the linear Kalman filter and the NEKF. Upper three panels show the truth signal (solid line), the filtered signal with the NEKF (thin dashed line) and the filtered signal with the linear Kalman filter (thick dashed-dotted line). The corresponding parameters of the observations are shown on top of each panel. The lower panel shows the profile of the fast forcing.

a large enough observation time $\Delta t$. Finally, we study the filter performance as a function of observation variance $r^{0}$. figure 3.8 shows the RMSE of filtering the two test trajectories. We note that the filter skill monotonically decreases as the observation variance increases. We also conclude that the observations of type 3 lead to the best filter skill and observations of type 1 lead to the worst filter skill among the three observation types. However, as was noted above, observations of types 2 and 3 lead to practically the same filter skill in the fast mode $u_{2}$. It is also worth noting that model error due to linearization is significantly larger in the case of the trajectory with strong fast forcing with $B=50$.

\section{Conclusions}

We started this paper by introducing the nonlinear multiple time test model with fast forcing. We motivated the model by the need of studying the effects of the moist convection, represented by the fast forcing, on the filtering atmospheric signals. We have shown a typical trajectory of the slow-fast system and discussed how the fast forcing impacts the dynamics of the amplitude of the fast mode. We then presented the analytical solution and first and second order statistics of the system. We specifically pointed out the non-Gaussianity of the statistics of the fast mode, which is driven by fast forcing and nonlinearly coupled to the slow mode. Then, we introduced the exact Nonlinear Extended Kalman Filter (NEKF) and the linear Kalman filter (KF) with model error for the slow-fast system with fast forcing. We compared the performance 

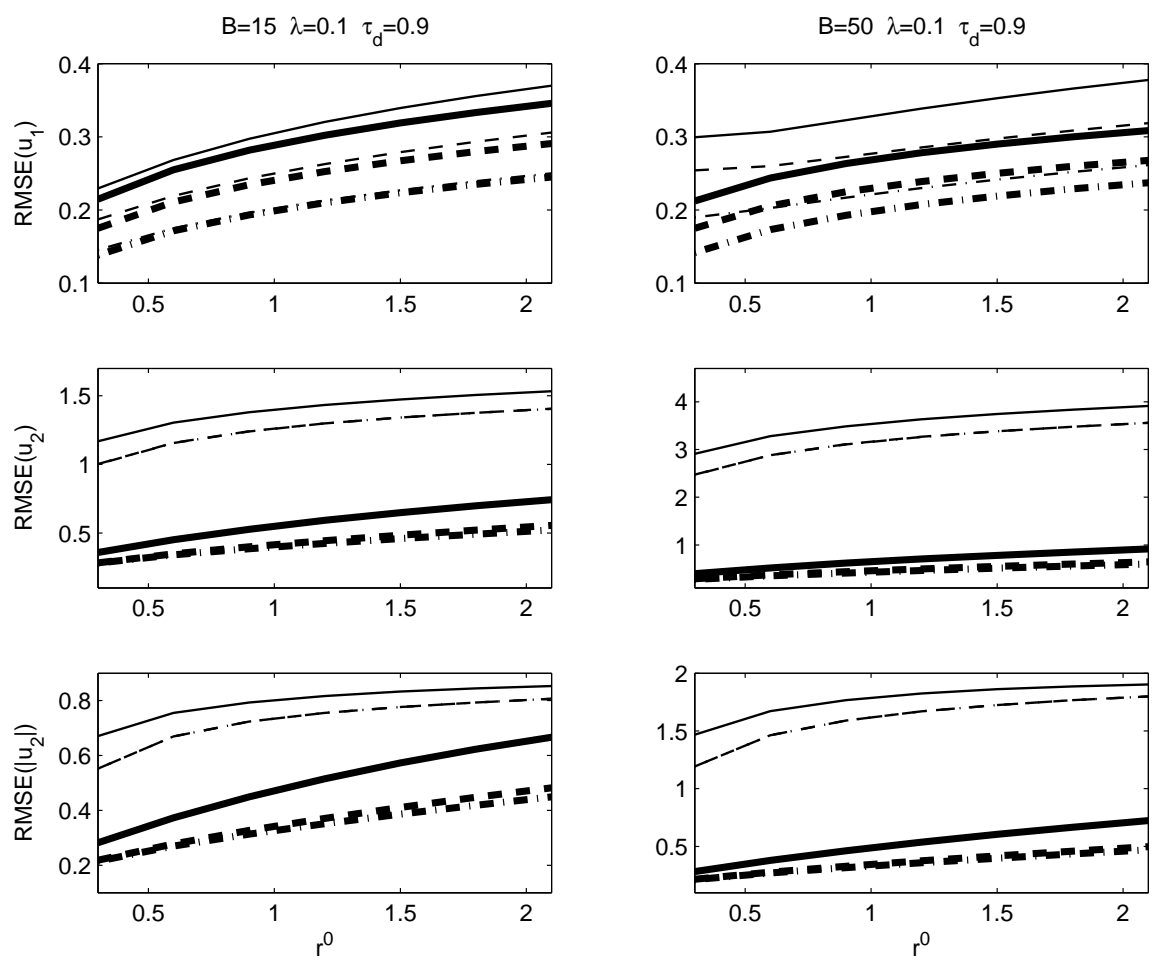

FIG. 3.8. RMSE as a function of observation variance $r^{0}$. The left column corresponds to the moderate fast forcing with $B=15$ and the right column corresponds to the strong fast forcing with $B=50$. The first row shows the RMSE of the slow mode, the second row shows the RMSE of the fast mode, and the third row shows the RMSE of the amplitude of the fast mode. Thick lines correspond to the exact NEKF and thin lines correspond to the linear Kalman filter. Solid lines correspond to observations of type 1, dashed lines correspond to observation of type 2, and dashed-dotted lines correspond to observations of type 3. The observation time was $\Delta t=0.2$.

of both filters under various filtering parameters. Next, we outline the results of our study of the filter performance.

We studied the deterioration of the filter skill around the observation times that were predicted by the observability analysis for the observations of types 1 and 2 . We also showed numerically that both the NEKF and the linear KF have high skill at the observation times that are away from those non-observable values. We note that the observations of type 3 have better skill for the slow mode than observations of types 2, which in turn lead to higher skill than observations of type 1. However, for the skill of the fast mode, observations of types 2 and 3 have almost equivalent skill which is significantly better than the skill with observations of type 1 . We have also discovered that for the observation time and observation variance larger than certain values and all three types of observations, the linear $\mathrm{KF}$ with model error performs surprisingly better than the exact NEKF on the slowly varying amplitude of the fast mode. This result should have practical significance when the envelope of the fast gravity waves driven by moist convection is an object of interest.

Acknowledgment. The research of A.J. Majda is partially supported by the National Science Foundation grant DMS-0456713, the Office of Naval Research grant 
N00014-05-1-0164, and the Defense Advanced Research Project Agency grant N0001407-1-0750. B. Gershgorin is supported as a postdoctoral fellow through the last two agencies.

Appendix A. In this Appendix, we compute the cross-covariance $\operatorname{Cov}\left(u_{2}, u_{1}\right)$, and $\operatorname{Cov}\left(u_{2}, u_{2}^{*}\right)$. We use the preliminary formulas for $\left\langle u_{20} e^{i b u_{10}}\right\rangle\left\langle u_{10} u_{20} e^{i b u_{10}}\right\rangle$ and $\left\langle u_{20}^{2} e^{i b u_{10}}\right\rangle$ that were obtained in [13]

$$
\begin{aligned}
& \left\langle u_{20} e^{i b u_{10}}\right\rangle \\
= & \left(\left\langle u_{20}\right\rangle+i b \operatorname{Cov}\left(u_{20}, u_{10}\right)\right) e^{i b\left\langle u_{10}\right\rangle-\frac{1}{2} \operatorname{Var}\left(u_{10}\right)}, \\
& \left\langle u_{10} u_{20} \exp \left(i b u_{10}\right)\right\rangle \\
= & \left(\left\langle u_{10}\right\rangle\left\langle u_{20}\right\rangle+\operatorname{Cov}\left(u_{20}, u_{10}\right)+i b\left(\left\langle u_{10}\right\rangle \operatorname{Cov}\left(u_{20}, u_{10}\right)+\left\langle u_{20}\right\rangle \operatorname{Var}\left(u_{10}\right)\right)\right. \\
& \left.-b^{2} \operatorname{Var}\left(u_{10}\right) \operatorname{Cov}\left(u_{20}, u_{10}\right)\right) \exp \left(i b\left\langle u_{10}\right\rangle-\frac{1}{2} b^{2} \operatorname{Var}\left(u_{10}\right)\right), \\
& \left\langle u_{20}^{2} \exp \left(i 2 b u_{10}\right)\right\rangle \\
= & \left(\operatorname{Cov}\left(u_{20}, u_{20}^{*}\right)+\left\langle u_{20}\right\rangle^{2}+4 b i\left\langle u_{20}\right\rangle \operatorname{Cov}\left(u_{20}, u_{10}\right)-4 b^{2} \operatorname{Cov}\left(u_{20}, u_{10}\right)^{2}\right) \\
& \times \exp \left(i 2 b\left\langle u_{10}\right\rangle-2 b^{2} \operatorname{Var}\left(u_{10}\right)\right) .
\end{aligned}
$$

A.1. Covariance $\operatorname{Cov}\left(u_{2}, u_{1}\right)$. Combining equations (2.7) and (2.9) we find

$$
\begin{aligned}
& \operatorname{Cov}\left(u_{2}, u_{1}\right)=\left\langle\left(u_{2}-\left\langle u_{2}\right\rangle\right)\left(u_{1}-\left\langle u_{1}\right\rangle\right)\right\rangle \\
= & \left\langle\left( e^{-\gamma_{2}\left(t-t_{0}\right)}\left[\psi\left(t_{0}, t\right) u_{20}-\left\langle\psi\left(t_{0}, t\right) u_{20}\right\rangle\right]+\int_{t_{0}}^{t} e^{-\gamma_{2}(t-s)}[\psi(s, t)-\langle\psi(s, t)\rangle] f_{2}(s) d s\right.\right. \\
& \left.\left.+\sigma_{2} \int_{t_{0}}^{t} e^{-\gamma_{2}(t-s)} \psi(s, t) d W_{2}(s)\right)\left(\left[u_{10}-\left\langle u_{10}\right\rangle\right] e^{-\gamma_{1}\left(t-t_{0}\right)}+\sigma_{1} \int_{t_{0}}^{t} e^{-\gamma_{1}(t-s)} d W_{1}(s)\right)\right\rangle \\
= & e^{-\left(\gamma_{1}+\gamma_{2}\right)\left(t-t_{0}\right)}\left[\left\langle u_{10} u_{20} \psi\left(t_{0}, t\right)\right\rangle-\left\langle u_{10}\right\rangle\left\langle u_{20} \psi\left(t_{0}, t\right)\right\rangle\right] \\
& +\int_{t_{0}}^{t} e^{-\gamma_{2}(t-s)}\left[\left\langle u_{10} \psi(s, t)\right\rangle-\left\langle u_{10}\right\rangle\langle\psi(s, t)\rangle\right] f_{2}(s) d s \\
& +\sigma_{1} e^{-\gamma_{2}\left(t-t_{0}\right)}\left\langle\int_{t_{0}}^{t} e^{-\gamma_{1}(t-s)} d W_{1}(s) u_{20} \psi\left(t_{0}, t\right)\right\rangle \\
& +\sigma_{1} \int_{t_{0}}^{t} e^{-\gamma_{2}(t-s)}\left\langle\psi(s, t) \int_{t_{0}}^{t} e^{-\gamma_{1}\left(t-s^{\prime}\right)} d W_{1}\left(s^{\prime}\right)\right\rangle f_{2}(s) d s .
\end{aligned}
$$

In the first term, we use

$$
\begin{aligned}
& \left\langle u_{10} u_{20} \psi\left(t_{0}, t\right)\right\rangle-\left\langle u_{10}\right\rangle\left\langle u_{20} \psi\left(t_{0}, t\right)\right\rangle \\
= & \left(\operatorname{Cov}\left(u_{20}, u_{10}\right)+i b\left(t_{0}, t\right)\left\langle u_{20}\right\rangle \operatorname{Var}\left(u_{10}\right)-b\left(t_{0}, t\right)^{2} \operatorname{Var}\left(u_{10}\right) \operatorname{Cov}\left(u_{20}, u_{10}\right)\right) \\
& \times \exp \left(i b\left(t_{0}, t\right)\left\langle u_{10}\right\rangle-\frac{1}{2} b\left(t_{0}, t\right)^{2} \operatorname{Var}\left(u_{10}\right)\right) \psi_{D}\left(t_{0}, t\right) \exp \left(-\frac{1}{2} \operatorname{Var}\left(J_{W}\left(t_{0}, t\right)\right)\right) .
\end{aligned}
$$


In the second term, we have

$$
\begin{aligned}
& \left\langle u_{10} \psi(s, t)\right\rangle-\left\langle u_{10}\right\rangle\langle\psi(s, t)\rangle \\
= & i \operatorname{Var}\left(u_{10}\right) b(s, t) \exp \left(i b(s, t)\left\langle u_{10}\right\rangle-\frac{1}{2} b(s, t)^{2} \operatorname{Var}\left(u_{10}\right)\right) \\
& \times \psi_{D}(s, t) \exp \left(-\frac{1}{2} \operatorname{Var}\left(J_{W}(s, t)\right)\right) .
\end{aligned}
$$

In order to compute the third and the fourth terms, we note that

$$
\begin{aligned}
& \frac{\partial}{\partial t}\left\langle\exp \left(i J_{W}(s, t)\right)\right\rangle=\frac{\partial}{\partial t}\left\langle\exp \left(i \int_{s}^{t} d s^{\prime} \sigma_{1} a_{0} \int_{t_{0}}^{s^{\prime}} e^{-\gamma_{1}\left(s^{\prime}-s^{\prime \prime}\right)} d W_{1}\left(s^{\prime \prime}\right)\right)\right\rangle \\
= & i \sigma_{1} a_{0}\left\langle\exp \left(i J_{W}(s, t)\right) \int_{t_{0}}^{t} e^{-\gamma_{1}\left(t-s^{\prime}\right)} d W_{1}\left(s^{\prime}\right)\right\rangle .
\end{aligned}
$$

On the other hand, we obtain

$$
\frac{\partial}{\partial t}\left\langle\exp \left(i J_{W}(s, t)\right)\right\rangle=-\frac{1}{2} \exp \left(-\frac{1}{2} \operatorname{Var}\left(J_{W}(s, t)\right)\right) \frac{\partial}{\partial t}\left(\operatorname{Var}\left(J_{W}(s, t)\right)\right),
$$

where

$$
\frac{\partial}{\partial t}\left(\operatorname{Var}\left(J_{W}(s, t)\right)\right)=\frac{\sigma_{1}^{2} a_{0}^{2}}{\gamma_{1}^{2}}\left(1+e^{-\gamma_{1}\left(s+t-2 t_{0}\right)}\left[-1-e^{2 \gamma_{1}\left(s-t_{0}\right)}+e^{\gamma_{1}(s-t)}\right]\right) .
$$

Then, after some simplifications, the third term becomes

$$
\begin{aligned}
& \sigma_{1} e^{-\gamma_{2}\left(t-t_{0}\right)}\left\langle u_{20} \psi\left(t_{0}, t\right) \int_{t_{0}}^{t} e^{-\gamma_{1}(t-s)} d W_{1}(s)\right\rangle \\
= & \frac{i}{2 a_{0}} e^{-\gamma_{2}\left(t-t_{0}\right)} \frac{\partial}{\partial t}\left(\operatorname{Var}\left(J_{W}\left(t_{0}, t\right)\right)\right)\left\langle u_{20} \psi\left(t_{0}, t\right)\right\rangle .
\end{aligned}
$$

The fourth part becomes

$$
\begin{aligned}
& \sigma_{1} \int_{t_{0}}^{t} e^{-\gamma_{2}(t-s)}\left\langle\psi(s, t) \int_{t_{0}}^{t} e^{-\gamma_{1}\left(t-s^{\prime}\right)} d W_{1}\left(s^{\prime}\right)\right\rangle f_{2}(s) d s \\
= & \frac{i}{2 a_{0}} \int_{t_{0}}^{t} e^{-\gamma_{2}(t-s)} \frac{\partial}{\partial t}\left(\operatorname{Var}\left(J_{W}(s, t)\right)\right)\langle\psi(s, t)\rangle f_{2}(s) d s .
\end{aligned}
$$

Finally, we obtain

$$
\begin{aligned}
& \operatorname{Cov}\left(u_{2}, u_{1}\right) \\
= & \left(\left[\operatorname{Cov}\left(u_{20}, u_{10}\right)+i b\left(t_{0}, t\right)\left\langle u_{20}\right\rangle \operatorname{Var}\left(u_{10}\right)\right.\right. \\
& \left.-b\left(t_{0}, t\right)^{2} \operatorname{Var}\left(u_{10}\right) \operatorname{Cov}\left(u_{20}, u_{10}\right)\right] e^{-\left(\gamma_{1}+\gamma_{2}\right)\left(t-t_{0}\right)}+\frac{i}{2 a_{0}} e^{-\gamma_{2}\left(t-t_{0}\right)} \\
& \left.\times \frac{\partial}{\partial t}\left(\operatorname{Var}\left(J_{W}\left(t_{0}, t\right)\right)\right)\left[\left\langle u_{20}\right\rangle+i \operatorname{Cov}\left(u_{20}, u_{10}\right) b\left(t_{0}, t\right)\right]\right) \\
& \times \psi_{D}\left(t_{0}, t\right) \exp \left(-\frac{1}{2} \operatorname{Var}\left(J_{W}\left(t_{0}, t\right)\right)\right) \exp \left(i b\left(t_{0}, t\right)\left\langle u_{10}\right\rangle-\frac{1}{2} b\left(t_{0}, t\right)^{2} \operatorname{Var}\left(u_{10}\right)\right) \\
+ & \frac{i}{2} \int_{t_{0}}^{t} e^{-\gamma_{2}(t-s)} f_{2}(s)\langle\psi(s, t)\rangle\left[2 \operatorname{Var}\left(u_{10}\right) b(s, t)+\frac{1}{a_{0}} \frac{\partial}{\partial t}\left(\operatorname{Var}\left(J_{W}(s, t)\right)\right)\right] d s .
\end{aligned}
$$


A.2. Covariance $\operatorname{Cov}\left(u_{2}, u_{2}^{*}\right)$. First we compute the correlator $\left\langle u_{2}^{2}\right\rangle$ by taking square of equation (2.9) and then averaging it

$$
\begin{aligned}
& \left\langle u_{2}^{2}\right\rangle \\
= & \left\langle\left( e^{-\gamma_{2}\left(t-t_{0}\right)} \psi\left(t_{0}, t\right) u_{20}+\int_{t_{0}}^{t} e^{-\gamma_{2}(t-s)} \psi(s, t) f_{2}(s) d s+\right.\right. \\
& \left.\sigma_{2} \int_{t_{0}}^{t} e^{-\gamma_{2}(t-s)} \psi(s, t) d W_{2}(s)\right)\left(e^{-\gamma_{2}\left(t-t_{0}\right)} \psi\left(t_{0}, t\right) u_{20}\right. \\
& \left.\left.+\int_{t_{0}}^{t} e^{-\gamma_{2}(t-s)} \psi(s, t) f_{2}(s) d s+\sigma_{2} \int_{t_{0}}^{t} e^{-\gamma_{2}(t-s)} \psi(s, t) d W_{2}(s)\right)\right\rangle \\
= & e^{-2 \gamma_{2}\left(t-t_{0}\right)}\left\langle u_{20}^{2} \psi^{2}\left(t, t_{0}\right)\right\rangle+\int_{t_{0}}^{t} d s \int_{t_{0}}^{t} d r e^{-\gamma_{2}(2 t-s-r)}\langle\psi(s, t) \psi(r, t)\rangle f_{2}(s) f_{2}(r) \\
& +2 e^{-\gamma_{2}\left(t-t_{0}\right)} \int_{t_{0}}^{t} e^{-\gamma_{2}(t-s)}\left\langle u_{20} \psi\left(t_{0}, t\right) \psi(s, t)\right\rangle f_{2}(s) d s .
\end{aligned}
$$

We compute the right hand side term by term

$$
\begin{aligned}
& e^{-2 \gamma_{2}\left(t-t_{0}\right)}\left\langle u_{20}^{2} \psi^{2}\left(t, t_{0}\right)\right\rangle \\
= & \exp \left(-2 \gamma_{2}\left(t-t_{0}\right)+2 i J_{D}\left(t_{0}, t\right)-2 \operatorname{Var}\left(J_{W}\left(t_{0}, t\right)\right)\right)\left\langle u_{20}^{2} \exp \left(i 2 b\left(t_{0}, t\right) u_{10}\right)\right\rangle .
\end{aligned}
$$

In order to compute the double integral, we first compute the following average

$$
\begin{aligned}
& \langle\psi(s, t) \psi(r, t)\rangle \\
= & e^{i\left(J_{D}(s, t)+J_{D}(r, t)\right)}\left\langle e^{i\left(J_{W}(s, t)+J_{W}(r, t)\right)}\right\rangle\left\langle e^{i(b(s, t)+b(r, t)) u_{10}}\right\rangle \\
= & e^{i\left(J_{D}(s, t)+J_{D}(r, t)\right)} e^{-\frac{1}{2} \operatorname{Var}\left(J_{W}(s, t)+J_{W}(r, t)\right)} e^{i(b(s, t)+b(r, t))\left\langle u_{10}\right\rangle-\frac{1}{2}(b(s, t)+b(r, t))^{2} \operatorname{Var}\left(u_{10}\right)},
\end{aligned}
$$

where

$$
\operatorname{Var}\left(J_{W}(s, t)+J_{W}(r, t)\right)=\operatorname{Var}\left(J_{W}(s, t)\right)+\operatorname{Var}\left(J_{W}(r, t)\right)+2 \operatorname{Cov}\left(J_{W}(s, t), J_{W}(r, t)\right) .
$$

Let us assume that $s<r$. Then, we find

$$
\begin{aligned}
\operatorname{Cov}\left(J_{W}(s, t), J_{W}(r, t)\right) & =\operatorname{Cov}\left(J_{W}(s, r)+J_{W}(r, t), J_{W}(r, t)\right) \\
& =\operatorname{Cov}\left(J_{W}(s, r), J_{W}(r, t)\right)+\operatorname{Var}\left(J_{W}(r, t)\right) .
\end{aligned}
$$

The covariance in the right hand side can be computed using the fact that intervals $(s, r)$ and $(r, t)$ do not intersect:

$$
\begin{aligned}
& \operatorname{Cov}\left(J_{W}(s, r), J_{W}(r, t)\right) \\
= & \sigma_{1}^{2} a_{0}^{2}\left\langle\int_{s}^{r} d s^{\prime} \int_{t_{0}}^{s^{\prime}} e^{\gamma_{1}\left(s^{\prime \prime}-s^{\prime}\right)} d W_{1}\left(s^{\prime \prime}\right) \int_{r}^{t} d r^{\prime} \int_{t_{0}}^{r^{\prime}} e^{\gamma_{1}\left(r^{\prime \prime}-r^{\prime}\right)} d W_{1}\left(r^{\prime \prime}\right)\right\rangle \\
= & \sigma_{1}^{2} a_{0}^{2} \int_{s}^{r} d s^{\prime} \int_{r}^{t} d r^{\prime} \int_{t_{0}}^{s^{\prime}} \exp \left(\gamma_{1}\left(2 s^{\prime \prime}-s^{\prime}-r^{\prime}\right)\right) d s^{\prime \prime} \\
= & -\frac{\sigma_{1}^{2} a_{0}^{2}}{2 \gamma_{1}^{3}}\left(1-e^{\gamma_{1}(s-r)}\right)\left(1-e^{\gamma_{1}(t-r)}\right)\left(e^{\gamma_{1}(r-t)}-e^{\gamma_{1}\left(2 t_{0}-s-t\right)}\right) .
\end{aligned}
$$


In the case when $s>r$ we have to swap $r$ and $s$ in the last expression. In order to obtain a general formula, we use $\min (s, r)$ instead of $s$ and $\max (s, r)$ instead of $r$ in the right hand side of equation (A.10) and in equation (A.11). Now, in order to compute the double integral in equation (A.9), we use the fact that $f_{2}$ has finite support

$$
\begin{aligned}
& \int_{t_{0}}^{t} d s \int_{t_{0}}^{t} d r e^{-\gamma_{2}(2 t-s-r)}\langle\psi(s, t) \psi(r, t)\rangle f_{2}(s) f_{2}(r) \\
= & \sum_{j} \sum_{m} \int_{I_{j}} d s \int_{I_{m}} d r f_{2}(s) f_{2}(r) e^{-\gamma_{2}(2 t-s-r)}\langle\psi(s, t) \psi(r, t)\rangle .
\end{aligned}
$$

Next, we compute the last term in the right hand side of equation (A.9). First, we find

$$
\left\langle u_{20} \psi\left(t_{0}, t\right) \psi(s, t)\right\rangle=e^{i\left(J_{D}\left(t_{0}, t\right)+J_{D}(s, t)\right)}\left\langle e^{i\left(J_{W}\left(t_{0}, t\right)+J_{W}(s, t)\right)}\right\rangle\left\langle u_{20} e^{i\left(b\left(t_{0}, t\right)+b(s, t)\right) u_{10}}\right\rangle,
$$

where

$$
\begin{aligned}
& \left\langle e^{i\left(J_{W}\left(t_{0}, t\right)+J_{W}(s, t)\right)}\right\rangle=e^{-\frac{1}{2} \operatorname{Var}\left(J_{W}\left(t_{0}, t\right)+J_{W}(s, t)\right)} \\
= & e^{-\frac{1}{2} \operatorname{Var}\left(J_{W}\left(t_{0}, t\right)\right)-\frac{3}{2} \operatorname{Var}\left(J_{W}(s, t)\right)-\operatorname{Cov}\left(J_{W}\left(t_{0}, s\right), J_{W}(s, t)\right)} .
\end{aligned}
$$

The covariance in equation (A.12) is computed using equation (A.11) and quadrature formula (2.14)

$$
\begin{aligned}
& \int_{t_{0}}^{t} e^{-\gamma_{2}(t-s)}\left\langle u_{20} \psi\left(t_{0}, t\right) \psi(s, t)\right\rangle f_{2}(s) d s=e^{i J_{D}\left(t_{0}, t\right)-\frac{1}{2} \operatorname{Var}\left(J_{W}\left(t_{0}, t\right)\right)} \\
& \times \sum_{j} \int_{I_{j}} f_{2}(s) e^{-\gamma_{2}(t-s)+i J_{D}(s, t)-\frac{3}{2} \operatorname{Var}\left(J_{W}(s, t)\right)-\operatorname{Cov}\left(J_{W}\left(t_{0}, s\right), J_{W}(s, t)\right)} \\
& \times\left\langle u_{20} e^{i\left(b\left(t_{0}, t\right)+b(s, t)\right) u_{10}}\right\rangle d s .
\end{aligned}
$$

Finally, to find the covariance $\operatorname{Cov}\left(u_{2}, u_{2}^{*}\right)$, we use

$$
\operatorname{Cov}\left(u_{2}, u_{2}^{*}\right)=\left\langle u_{2}^{2}\right\rangle-\left\langle u_{2}\right\rangle^{2}
$$

\section{REFERENCES}

[1] B.D.O. Anderson and J.B. Moore, Optimal Filtering, Prentice-Hall Englewood Cliffs, NJ, 1979

[2] J.L. Anderson, An ensemble adjustment Kalman filter for data assimilation, Mon. Wea. Rev., 129(12), 2884-2903, 2001.

[3] J.L. Anderson, A local least squares framework for ensemble filtering, Mon. Wea. Rev., 131(4), 634-642, 2003.

[4] E. Castronovo, J. Harlim and A. Majda, Mathematical criteria for filtering complex systems: plentiful observations, J. Comp. Phys., 227(7), 3678-3714, 2008.

[5] C. Chui and G. Chen, Kalman Filtering, Springer New York, 1999.

[6] S.E. Cohn and S.F. Parrish, The behavior of forecast error covariances for a Kalman filter in two dimensions, Mon. Wea. Rev., 119, 1757-1785, 1991.

7] R. Daley, Atmospheric Data Analysis, Cambridge University Press, 457, 1991.

[8] R. Durrett, Stochastic Calculus: a Practical Introduction, CRC-Press LLC, 1996.

[9] P.F. Embid and A.J. Majda, Low Froude number limiting dynamics for stably stratified flow with small or fixed Rossby Number, Geophys. Astrophys. Fluid Dyn., 87, 1-50, 1998.

[10] G. Evensen, Sequential data assimilation with a nonlinear quasigeostrophic model using Monte Carlo methods to forecast error statistics, J. Geophys. Res., 99, 10143-10162, 1994. 
[11] G. Evensen, Advanced data assimilation for strongly nonlinear dynamics, Mon. Wea. Rev., $125,1342-1354,1997$.

[12] C.W. Gardiner Handbook of Stochastic Methods for Physics, Chemistry, and Natural Sciences, Springer-Verlag, New York, 1997.

[13] B. Gershgorin and A. Majda, A nonlinear test model for filtering slow-fast systems, Commun. Math. Sci., 6, 611-649, 2008.

[14] M. Ghil and P. Malanotte-Rizzoli, Data assimilation in meteorology and oceanography, Adv. Geophys., 33, 141-266, 1991.

[15] J. Harlim and A. Majda, Mathematical strategies for filtering complex systems: regularly spaced sparse observations, J. Comp. Phys, doi:10.1016/j.jcp2008.01.049, 2008.

[16] J. Harlim and A. Majda, Filtering nonlinear dynamical systems with linear stochastic models, Nonlinearity, submitted, 2007.

[17] J. Harlim and A. Majda, Catastrophic filter divergence in filtering nonlinear dissipative systems, Commun. Math. Sci., to appear, 2009.

[18] R. Klein and A. Majda, Systematic multiscale models for deep convection on mesoscales, Theo. Comp. Fluid Dyn., 20, 525-551, 2006.

[19] R. Kubo, Stochastic Liouville equations, J. Math. Phys., 4(2), 174-183, 1963.

[20] A. Majda, Introduction to PDEs and Waves for the Atmosphere and Ocean, Courant Lecture Notes in Mathematics, AIMS/CIMS, New York, 9, 2003.

[21] A.J. Majda and M. Grote, Explicit off-line criteria for stable accurate time filtering of strongly unstable spatially extended systems, Proc. Nat. Acad. Sci., 104, 1124-1129, 2007.

[22] A.J. Majda and P.R. Kramer, Simplified models for turbulent diffusion: theory, numerical modelling and physical phenomena, Phys. Rep., 314(4-5), 237-574, 1999.

[23] A.J. Majda, I. Timofeyev and E. Vanden-Eijnden, A mathematical framework for stochastic climate models, Commun. Pure Appl. Math., 54, 891-974, 2001.

[24] A.J. Majda and B. Khouider, A simple multicloud parameterization for convectively coupled tropical waves. Part I: linear analysis, J. Atmos. Sci., 63(4), 1308-1323, 2006.

[25] A.J. Majda and B. Khouider, Stochastic and mesoscopic models for tropical convection, PNAS, 99(3), 1123-1128, 2002.

[26] A.J. Majda, I. Timofeyev and E. Vanden-Eijnden, A priori tests of a stochastic mode reduction strategy, Physica D, 170, 206-252, 2002.

[27] E. Ott, B. Hunt, I. Szunyogh, A. Zimin, E. Kostelich, M. Corrazza, E. Kalnay and J. Yorke, A local ensemble Kalman filter for atmospheric data assimilation, Tellus A, 56, 415-428, 2004.

[28] R. Salmon, Lectures on Geophysical Fluid Dynamics, Oxford university Press, 378, 1998.

[29] A. Simmons, The control of gravity waves in data assimilation, ECMWF, 1999.

[30] L.M. Smith, Numerical study of two-dimensional stratified turbulence. Advances in wave interaction and turbulence, Contemp. Math., 283, 2001.

[31] L.M. Smith and F. Waleffe, Generation of slow large scales in forced rotating stratified turbulence, J. Fluid Mech., 451, 145-168, 2002.

[32] M.L. Waite and P. Bartello, Stratified turbulence dominated by vortical motion, J. Fluid Mech., 517, 281-308, 2004. 